\title{
On the Local Well-Posedness of the Cauchy Problem for a Modified Two-Component Camassa-Holm System in Besov Spaces
}

\author{
Jiangbo Zhou, ${ }^{1}$ Lu Yao, ${ }^{1}$ Lixin Tian, ${ }^{1}$ and Wenbin Zhang ${ }^{2}$ \\ ${ }^{1}$ Department of Mathematics, Jiangsu University, Zhenjiang, Jiangsu 212013, China \\ ${ }^{2}$ Taizhou Institute of Science and Technology, NUST, Taizhou, Jiangsu 225300, China \\ Correspondence should be addressed to Jiangbo Zhou; ujszjb@126.com
}

Received 26 April 2013; Accepted 14 November 2013

Academic Editor: Athanasios N. Yannacopoulos

Copyright (C) 2013 Jiangbo Zhou et al. This is an open access article distributed under the Creative Commons Attribution License, which permits unrestricted use, distribution, and reproduction in any medium, provided the original work is properly cited.

We consider the Cauchy problem for an integrable modified two-component Camassa-Holm system with cubic nonlinearity. By using the Littlewood-Paley decomposition, nonhomogeneous Besov spaces, and a priori estimates for linear transport equation, we prove that the Cauchy problem is locally well-posed in Besov spaces $B_{p, r}^{s}$ with $1 \leq p, r \leq+\infty$ and $s>\max \{2+(1 / p), 5 / 2\}$.

\section{Introduction}

The following modified Camassa-Holm equation with cubic nonlinearity

$$
m_{t}+\left(\left(u^{2}-u_{x}^{2}\right) m\right)_{x}=0, \quad m=u-u_{x x}
$$

was proposed as a integrable system by Fuchssteiner [1] and Olver and Rosenau [2] by applying the general method of tri-Hamiltonian duality to the bi-Hamiltonian representation of the modified Korteweg-de Vries equation. Later, it was obtained by Qiao [3] from the two-dimensional Euler equations, where the variables $u(t, x)$ and $m(t, x)$ represent, respectively, the velocity of the fluid and its potential density. Qiao also [3] obtained the cuspons and $W / M$-shape-peaks solitons of (1). In [4], it was shown that (1) admits a Lax pair and hence can be solved by the method of inverse scattering. Fu et al. [5] showed that the Cauchy problem of (1) is locally well-posed in a range of the Besov spaces. They determined the blow-up scenario and the lower bound of the maximal time of existence. They also described a blow-up mechanism for solutions with certain initial profiles and the nonexistence of the smooth traveling wave solutions was also demonstrated. In addition, they obtained the persistence properties of the strong solutions for (1). Gui et al. [6] investigated the formation of singularities and showed that singularities of the solutions occur only in the form of wave breaking. They obtained a new wave-breaking mechanism for solutions with certain initial profiles. It was proved that the peaked functions of the form

$$
u(t, x)= \pm \sqrt{\frac{3 c}{2}} e^{-|x-c t|}, \quad c \in \mathbb{R}^{+}
$$

are global weak solutions to (1) [6].

Recently, Song et al. [7] suggested a new integrable twocomponent vision of (1) as follows:

$$
\begin{aligned}
m_{t} & =[m a(u, v)]_{x}, \\
h_{t} & =[h a(u, v)]_{x},
\end{aligned}
$$

with $m=u-u_{x x}, h=v-v_{x x}$, and $a(u, v)=u_{x} v_{x}-u v+$ $u v_{x}-u_{x} v$. Apparently, it reduces to (1) when $v=u$. They showed that this system has Lax-pair and is also geometrically integrable. As a consequence of geometric integrability, its conservation laws were constructed by expanding the pseudopotential. Finally, the cuspons and $W / M$-shape solitons of system (3) were obtained. 
In this paper, we are interesting in the local well-posedness of the following Cauchy problem for (3)

$$
\begin{gathered}
m_{t}=[m a(u, v)]_{x}, \quad x \in \mathbb{R}, t \geq 0, \\
h_{t}=[h a(u, v)]_{x}, \quad x \in \mathbb{R}, t \geq 0, \\
u(0, x)=u_{0}(x), \quad v(0, x)=v_{0}(x), \quad x \in \mathbb{R},
\end{gathered}
$$

with $m=u-u_{x x}, h=v-v_{x x}$, and $a(u, v)=u_{x} v_{x}-u v+$ $u v_{x}-u_{x} v$. Although Kato's theory is a useful method to obtain the local well-posedness of the Cauchy problem in Sobolev space $H^{s}(\mathbb{R})$ for lots of equations, such as the CamassaHolm equation [8], the Degasperis-Procesi equation [9], and the Novikov equation [10]. However, it seems to be unapplicable to the Cauchy problem (4). Fortunately, by using the Littlewood-Paley decomposition and nonhomogeneous Besov spaces, we prove that the Cauchy problem (4) is locally well-posed in the Besov space $B_{p, r}^{s}$ with $1 \leq p, r \leq+\infty$, and $s>\max \{1+(1 / p), 5 / 2\}$. As a corollary, it is locally well-posed in the Sobolev space $H^{s}(\mathbb{R})$ with $s>5 / 2$. The LittlewoodPaley decomposition and nonhomogeneous Besov spaces which were introduced in [11] have been used to establish the well-posedness of the Euler equations, the NavierStokes equations, the Camassa-Holm equation, the Novikov equation, the two-component Camassa-Holm system, the two-component Degasperis-Procesi system, and the coupled Camassa-Holm equations; see [12-20].

The rest of the paper is organized as follows. In Section 2, we recall some basic results on Besov spaces and linear transport equation. In Section 3, we establish the local wellposedness of the Cauchy problem (4) in a range of Besov spaces. Some open problems are presented in Section 4.

Notation. Since all spaces of functions are over $\mathbb{R}$, we drop $\mathbb{R}$ in our notations of function spaces for simplicity. For a given Banach space $E$, we denote its norm by $\|\cdot\|_{E}$. We denote $\mathscr{F} u$ or $\widehat{u}$ the Fourier transform of the function $u$. Let $\mathscr{C}^{k}([0, T] ; H)$ denote the space of all $k$ times continuously differentiable functions $u(x, t)$ defined on $[0, t]$ with values in space $H$. $L^{\infty}([0, T] ; H)$ denotes the space of functions $u(x, t)$ which belong to $H$ as a function of $x$ for every fixed $t(0 \leq t \leq T)$ and $\sup _{0 \leq t \leq T}\|u(\cdot, t)\|_{H}<\infty$ and $L^{1}([0, T] ; H)$ the space of functions $u(x, t)$ which belong to $H$ as a function of $x$ for every fixed $t(0 \leq t \leq T)$ and $\int_{0}^{T}\|u(\cdot, t)\|_{H} d t<\infty$. $\mathcal{S}^{\prime}$ denotes the tempered distribution spaces.

\section{Preliminaries}

In this section, we recall some results on the Littlewood-Paley decomposition, the nonhomogeneous Besov spaces, and the linear transport equation which can be seen in [10-20].

Proposition 1 (Littlewood-Paley decomposition). Let $\mathscr{B} \triangleq$ $\{\xi \in \mathbb{R},|\xi| \leq 4 / 3\}$ and $\mathscr{C} \triangleq\{\xi \in \mathbb{R}, 3 / 4 \leq|\xi| \leq 8 / 3\}$. There exist two radial functions $\chi \in \mathscr{C}_{c}^{\infty}(\mathscr{B})$ and $\varphi \in \mathscr{C}_{c}^{\infty}(\mathscr{C})$ such that

$$
\chi(\xi)+\sum_{q \geq 0} \varphi\left(2^{-q} \xi\right)=1, \quad \forall \xi \in \mathbb{R}^{d}
$$

$$
\begin{gathered}
\left|q-q^{\prime}\right| \geq 2 \Longrightarrow \operatorname{Supp} \varphi\left(2^{-q} \cdot\right) \cap \operatorname{Supp} \varphi\left(2^{-q^{\prime}} \cdot\right)=\phi, \\
q \geq 1 \Longrightarrow \operatorname{Supp} \chi(\cdot) \cap \operatorname{Supp} \varphi\left(2^{-q} \cdot\right)=\phi, \\
\frac{1}{3} \leq \chi(\xi)^{2}+\sum_{q \geq 0} \varphi\left(2^{-q} \xi\right)^{2} \leq 1, \quad \forall \xi \in \mathbb{R}^{d} .
\end{gathered}
$$

Furthermore, let $h \triangleq \mathscr{F} \varphi, \widetilde{h} \triangleq \mathscr{F}^{-1} \chi$ and define the dyadic operators $\Delta_{q}$ and low-frequency cut-offoperators $S_{q}$ as follows:

$$
\begin{aligned}
\Delta_{q} f & \triangleq \varphi\left(2^{-q} D\right) f \\
& =2^{q d} \int_{\mathbb{R}^{d}} h\left(2^{q} y\right) f(x-y) d y, \quad \text { for } q \geq 0, \\
& \Delta_{-1} f \triangleq S_{0} f, \quad \Delta_{q} f \triangleq 0, \quad \text { for } q \leq-2, \\
S_{q} f & \triangleq \chi\left(2^{-q} D\right) f \\
& =\sum_{-1 \leq k \leq q-1} \Delta_{k} f=2^{q d} \int_{\mathbb{R}^{d}} \tilde{h}\left(2^{q} y\right) f(x-y) d y .
\end{aligned}
$$

Then, for all $u \in \mathcal{S}^{\prime}$, the nonhomogeneous Littlewood-Paley decomposition of a distribution $u$ is defined

$$
u=\sum_{q \in \mathbb{Z}} \Delta_{q} u
$$

Definition 2 (Besov space). Let $s \in \mathbb{R}$ and $1 \leq p, r \leq \infty$. The inhomogenous Besov space $B_{p, r}^{s}(\mathbb{R})\left(B_{p, r}^{s}\right.$ for short) is defined by

$$
B_{p, r}^{s} \triangleq\left\{f \in \mathcal{S}^{\prime}(\mathbb{R}),\|f\|_{B_{p, r}^{s}}<\infty\right\}
$$

where

$$
\|f\|_{B_{p, r}^{s}} \triangleq \begin{cases}\left(\sum_{q \in \mathbb{Z}} 2^{q s r}\left\|_{q} f\right\|_{L^{p}}^{r}\right)^{1 / r}, & \text { for } r<\infty, \\ \sup _{q \in \mathbb{Z}} 2^{q s}\left\|\Delta_{q} f\right\|_{L^{p}}, & \text { for } r=\infty .\end{cases}
$$

If $s=\infty, B_{p, r}^{\infty} \triangleq \bigcap_{s \in \mathbb{R}} B_{p, r}^{s}$.

Proposition 3 (properties of Besov space). Let $s \in \mathbb{R}$ and $1 \leq p, r, p_{j}, r_{j} \leq \infty, j=1,2$; then the following properties hold.

(1) Topological properties: $B_{p, r}^{s}$ is a Banach space which is continuously embedded in $\mathcal{S}^{\prime}$.

(2) Density: $\mathscr{C}_{c}^{\infty}$ is dense in $B_{p, r}^{s} \Leftrightarrow 1 \leq p, r<\infty$.

(3) Sobolev embeddings: if $p_{1} \leq p_{2}$ and $r_{1} \leq r_{2}$, then $B_{p_{1}, r_{1}}^{s} \hookrightarrow B_{p_{2}, r_{2}}^{s-d\left(\left(1 / p_{1}\right)-\left(1 / p_{2}\right)\right)}$. If $s_{1}<s_{2}, 1 \leq p \leq+\infty$ and $1 \leq r_{1}, r_{2} \leq+\infty$, then the embedding $B_{p, r_{2}}^{s_{2}} \hookrightarrow B_{p, r_{1}}^{s_{1}}$ is locally compact.

(4) Algebraic properties: for $s>0, B_{p, r}^{s} \cap L^{\infty}$ is an algebra. Moreover, $B_{p, r}^{s}$ is an algebra $\Leftrightarrow B_{p, r}^{s} \hookrightarrow L^{\infty} \Leftrightarrow s>d / p$ or $(s \geq$ $d / p$ and $r=1$ ). 
(5) Fatou property: if $\left(u^{(n)}\right)_{n \in \mathbb{N}}$ is a bounded sequence of $B_{p, r}^{s}$ which tends to $u$ in $\mathcal{S}^{\prime}$, then $u \in B_{p, r}^{s}$ and

$$
\|u\|_{B_{p, r}^{s}} \leq \liminf _{n \rightarrow \infty}\left\|u^{(n)}\right\|_{B_{p, r}^{s}} .
$$

(6) Complex interpolation: if $u \in B_{p, r}^{s} \cap B_{p, r}^{\widetilde{s}}$ and $\theta \in[0,1]$, $1 \leq p, r \leq \infty$, then $u \in B_{p, r}^{\theta s+(1-\theta) \widetilde{s}}$ and

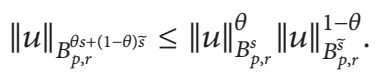

(7) Generalized derivatives: let $f \in \mathscr{C}^{\infty}\left(\mathbb{R}^{d}\right)$ and $f$ be a homogeneous function of degree $m \in \mathbb{R}$ away from a neighborhood of the origin. There exists a constant $C$ depending only on $f$ and such that

$$
\|f(D) u\|_{B_{p, r}^{s-m}} \leq C\|u\|_{B_{p, r}^{s}} .
$$

(8) 1-D Moser-type estimates:

(i) for $s>0$,

$$
\|f g\|_{B_{p, r}^{s}} \leq C\left(\|f\|_{B_{p, r}^{s}}\|g\|_{L^{\infty}}+\|g\|_{B_{p, r}^{s}}\|f\|_{L^{\infty}}\right),
$$

(ii) for $s_{1} \leq 1 / p, s_{2}>(1 / p)\left(s_{2} \geq 1 / p\right.$ if $\left.r=1\right)$ and $s_{1}+s_{2}>0$,

$$
\|f g\|_{B_{p, r}^{s_{1}}} \leq C\|f\|_{B_{p, r}^{s_{1}}}\|g\|_{B_{p, r}^{s_{2}}}
$$

where $C^{\prime}$ s are constants independent of $f$ and $g$.

Lemma 4 (a prior estimates for 1-D linear transport equation). Let $1 \leq p, r \leq \infty$, and $s>-\min \{1 / p, 1-(1 / p)\}$. Assume that $f_{0} \in B_{p, r}^{s}, F \in L^{1}\left([0, T] ; B_{p, r}^{s}\right)$ and $\partial_{x} v \in$ $L^{1}\left([0, T] ; B_{p, r}^{s-1}\right)$ if $s>1+(1 / p)$ or $\partial_{x} v \in L^{1}\left(0, T ; B_{p, r}^{1 / p} \cap L^{\infty}\right)$ otherwise. If $f \in L^{\infty}\left([0, T] ; B_{p, r}^{s}\right) \cap \mathscr{C}\left([0, T] ; \mathcal{S}^{\prime}\right)$ solves the following 1-D linear transport equation

$$
\begin{aligned}
& f_{t}+v f_{x}=F, \\
& f(x, 0)=f_{0},
\end{aligned}
$$

then there exists a constant $C$ depending only on $s, p$, and $r$ such that the following statements hold:

(1) If $r=1$ or $s \neq 1+(1 / p)$,

$$
\begin{aligned}
\|f\|_{B_{p, r}^{s}} \leq & \left\|f_{0}\right\|_{B_{p, r}^{s}}+\int_{0}^{t}\|F(\tau)\|_{B_{p, r}^{s}} d \tau \\
& +C \int_{0}^{t} V^{\prime}(\tau)\|f(\tau)\|_{B_{p, r}^{s}} d \tau
\end{aligned}
$$

or

$$
\|f\|_{B_{p, r}^{s}} \leq e^{C V(t)}\left(\left\|f_{0}\right\|_{B_{p, r}^{s}}+\int_{0}^{t} e^{-C V(\tau)}\|F(\tau)\|_{B_{p, r}^{s}} d \tau\right),
$$

where

$$
V(t)= \begin{cases}\int_{0}^{t}\left\|v_{x}(\tau)\right\|_{B_{p, r}^{1 / p} \cap L^{\infty}} d \tau, & \text { if } s<1+\frac{1}{p} \\ \int_{0}^{t}\left\|v_{x}(\tau)\right\|_{B_{p, r}^{s-1}} d \tau, & \text { if } s>1+\frac{1}{p} .\end{cases}
$$

(2) If $s \leq 1+(1 / p), f_{0, x} \in L^{\infty}((0, T) \times \mathbb{R})$, and $F_{x} \in$ $L^{1}\left([0, T] ; L^{\infty}\right)$, then

$$
\begin{aligned}
& \|f(t)\|_{B_{p, r}^{s}}+\left\|f_{x}(t)\right\|_{L^{\infty}} \\
& \leq e^{C V(t)}\left(\left\|f_{0}\right\|_{B_{p, r}^{s}}+\left\|f_{0, x}\right\|_{L^{\infty}}\right. \\
& \left.\quad+\int_{0}^{t} e^{-C V(\tau)}\left(\|F(\tau)\|_{B_{p, r}^{s}}+\left\|F_{x}(\tau)\right\|_{L^{\infty}}\right) d \tau\right),
\end{aligned}
$$

with $V(t)=\int_{0}^{t}\left\|v_{x}(\tau)\right\|_{B_{p, r}^{1 / p} \cap L^{\infty}} d \tau$.

(3) If $f=v$, then, for all $s>0$, estimate (17) holds true with $V(t)=\int_{0}^{t}\left\|v_{x}(\tau)\right\|_{L^{\infty}} d \tau$.

(4) If $r<\infty$, then $f \in \mathscr{C}\left([0, T] ; B_{p, r}^{s}\right)$.

(5) If $r=\infty$, then $f \in \mathscr{C}\left([0, T] ; B_{p, 1}^{s^{\prime}}\right)$ for all $s^{\prime}<s$.

Lemma 5 (existence and uniqueness). Let $p, r, s, f_{0}$, and $F$ be as in the statement of Lemma 4. Assume that $v \in$ $L^{\rho}\left([0, T] ; B_{\infty, \infty}^{-M}\right)$ for some $\rho>1$ and $M>0$ and $v_{x} \in$ $L^{1}\left([0, T] ; B_{p, r}^{s-1}\right)$ if $s>1+(1 / p)$ or $s=1+(1 / p)$ and $r=1$ and $v_{x} \in L^{1}\left([0, T] ; B_{p, r}^{1 / p} \cap L^{\infty}\right)$ if $s<1+(1 / p)$. Then the problem (15) has a unique solution $f \in L^{\infty}\left([0, T] ; B_{p, r}^{s}\right) \cap$ $\left(\bigcap_{s^{\prime}<s} \mathscr{C}\left([0, T] ; B_{p, 1}^{s^{\prime}}\right)\right)$ and the inequalities of Lemma 4 can hold true. Moreover, if $r<+\infty$, then $f \in \mathscr{C}\left([0, T] ; B_{p, r}^{s}\right)$.

\section{Local Well-Posedness}

In this section, we discuss the local well-posedness of the Cauchy problem (4).

For $T>0, s \in \mathbb{R}$, and $1 \leq p \leq \infty$, we denote

$$
\begin{aligned}
& E_{p, r}^{s}(T) \triangleq \mathscr{C}\left([0, T] ; B_{p, r}^{s}\right) \\
& \bigcap \mathscr{C}^{1}\left([0, T] ; B_{p, r}^{s-1}\right), \quad \text { for } 1 \leq r<\infty, \\
& E_{p, \infty}^{s}(T) \triangleq L^{\infty}\left([0, T] ; B_{p, \infty}^{s}\right) \bigcap \operatorname{Lip}\left([0, T] ; B_{p, \infty}^{s-1}\right) .
\end{aligned}
$$

Our main local well-posedness result is the following theorem.

Theorem 6. Suppose that $1 \leq p, r \leq+\infty, s>\max \{2+(1 / p)$, $(5 / 2)\}$, and $\left(u_{0}, v_{0}\right) \in B_{p, r}^{s} \times B_{p, r}^{s}$. Then, there exists a time $T>0$ such that the Cauchy problem (4) has a unique solution $(u, v) \in$ $E_{p, r}^{s}(T)^{2}$ and the map $\left(u_{0}, v_{0}\right) \rightarrow(u, v)$ is continuous from a neighborhood of $\left(u_{0}, v_{0}\right)$ in $B_{p, r}^{s} \times B_{p, r}^{s}$ into $E_{p, r}^{s^{\prime}}(T)^{2}$ for every $s^{\prime}<s$ when $r=+\infty$ and $s^{\prime}=s$ whereas $r<+\infty$.

Proof. We divide the proof into seven steps. In the following, we denote $C>0$ a generic constant only depending on $p, r$, and $s$.

Step 1 (approximate solution). We use a standard iterative process to construct a solution. Starting from $u_{0}:=0, v_{0}:=0$ 
by induction, we define a sequence of smooth functions $\left(u^{(n)}, v^{(n)}\right)_{n \in \mathbb{N}} \in \mathscr{C}\left(\mathbb{R}^{+} ; B_{p, r}^{\infty}\right)^{2}$ by solving the following linear transport equations:

$$
\begin{gathered}
\left\{\partial_{t}+\left[-a\left(u^{(n)}, v^{(n)}\right)\right] \partial_{x}\right\} m^{(n+1)}(t)=m^{(n)}\left[a\left(u^{(n)}, v^{(n)}\right)\right]_{x} \\
\left\{\partial_{t}+\left[-a\left(u^{(n)}, v^{(n)}\right)\right] \partial_{x}\right\} h^{(n+1)}(t)=h^{(n)}\left[a\left(u^{(n)}, v^{(n)}\right)\right]_{x} \\
\left.u^{(n+1)}\right|_{t=0}=u_{0}^{(n+1)}=S_{n+1} u_{0}, \\
\left.v^{(n+1)}\right|_{t=0}=v_{0}^{(n+1)}=S_{n+1} v_{0},
\end{gathered}
$$

with $a\left(u^{(n)}, v^{(n)}\right)=u_{x}^{(n)} v_{x}^{(n)}-u^{(n)} v^{(n)}+u^{(n)} v_{x}^{(n)}-u_{x}^{(n)} v^{(n)}$.

Since all the data belong to $B_{p, r}^{\infty}$, Lemma 5 enables us to show by induction that, for all $n \in \mathbb{N}$, system (21) has a global solution which belongs to $\mathscr{C}\left(\mathbb{R}^{+} ; B_{p, r}^{\infty}\right)^{2}$.

Step 2 (uniform bounds). Applying (17) to the first equation in (21) we have

$$
\begin{aligned}
\left\|m^{(n+1)}(t)\right\|_{B_{p, r}^{s-2}} \leq & \left\|m_{0}\right\|_{B_{p, r}^{s-2}} e^{C \int_{0}^{t}\left\|\partial_{x}\left[-a\left(u^{(n)}, v^{(n)}\right)\right](\tau)\right\|_{B_{, r, r}^{s-3}} d \tau} \\
+ & \int_{0}^{t} e^{C \int_{\tau}^{t}\left\|\partial_{x}\left[-a\left(u^{(n)}, v^{(n)}\right)\right]\left(\tau^{\prime}\right)\right\|_{B_{p, r}^{s-3}} d \tau^{\prime}} \\
& \times\left\|m^{(n)}\left[a\left(u^{(n)}, v^{(n)}\right)\right]_{x}(\tau)\right\|_{B_{p, r}^{s-2}} d \tau .
\end{aligned}
$$

Since $B_{p, r}^{s-2}$ is an algebra when $s>\max \{2+(1 / p), 5 / 2\}$, we derive from Proposition 3(7) that

$$
\begin{aligned}
\| \partial_{x}[ & \left.-a\left(u^{(n)}, v^{(n)}\right)\right] \|_{B_{p, r}^{s-3}} \\
& \leq C\left\|a\left(u^{(n)}, v^{(n)}\right)\right\|_{B_{p, r}^{s-2}} \\
& =C\left\|u_{x}^{(n)} v_{x}^{(n)}-u^{(n)} v^{(n)}+u^{(n)} v_{x}^{(n)}-u_{x}^{(n)} v^{(n)}\right\|_{B_{p, r}^{s-2}} \\
& \leq C\left\|u^{(n)}\right\|_{B_{p, r}^{s}}\left\|v^{(n)}\right\|_{B_{p, r}^{s}} \\
& \leq C\left(\left\|u^{(n)}\right\|_{B_{p, r}^{s}}+\left\|v^{(n)}\right\|_{B_{p, r}^{s}}\right)^{2}, \\
\left\|m^{(n)}\left[a\left(u^{(n)}, v^{(n)}\right)\right]_{x}\right\|_{B_{p, r}^{s-2}} & \leq C\left\|m^{(n)}\right\|_{B_{p, r}^{s-2}}\left\|\left[a\left(u^{(n)}, v^{(n)}\right)\right]_{x}\right\|_{B_{p, r}^{s-2}} \\
& \leq C\left\|m^{(n)}\right\|_{B_{p, r}^{s-2}}\left\|a\left(u^{(n)}, v^{(n)}\right)\right\|_{B_{p, r}^{s-1}} \\
& =C\left\|m^{(n)}\right\|_{B_{p, r}^{s-1}}\left\|u_{x}^{(n)} v_{x}^{(n)}-u^{(n)} v^{(n)}+u^{(n)} v_{x}^{(n)}-u_{x}^{(n)} v^{(n)}\right\|_{B_{p, r}^{s-1}} \\
& \leq C\left\|u^{(n)}\right\|_{B_{p, r}^{s}}\left\|u^{(n)}\right\|_{B_{p, r}^{s}}\left\|v^{(n)}\right\|_{B_{p, r}^{s}} \\
& \leq C\left\|u^{(n)}\right\|_{B_{p, r}^{s}}^{2}\left\|v^{(n)}\right\|_{B_{p, r}^{s}} .
\end{aligned}
$$

It follows from (22)-(23) that

$$
\begin{aligned}
& \left\|u^{(n+1)}(t)\right\|_{B_{p, r}^{s}} \leq\left\|u_{0}\right\|_{B_{p, r}^{s}} e^{C \int_{0}^{t}\left(\left\|u^{(n)}(\tau)\right\|_{p_{p, r}^{s}}+\left\|v^{(n)}(\tau)\right\|_{B_{p, r}^{s}}\right)^{2} d \tau} \\
& +C \int_{0}^{t} e^{C \int_{\tau}^{t}\left(\left\|u^{(n)}\left(\tau^{\prime}\right)\right\|_{p, r}^{s}+\left\|v^{(n)}\left(\tau^{\prime}\right)\right\|_{B_{p, r}^{s}}\right)^{2} d \tau^{\prime}} \\
& \times\left\|u^{(n)}(\tau)\right\|_{B_{p, r}^{s}}^{2}\left\|v^{(n)}(\tau)\right\|_{B_{p, r}^{s}} d \tau .
\end{aligned}
$$

Similarly, we have

$$
\begin{aligned}
&\left\|v^{(n+1)}(t)\right\|_{B_{p, r}^{s}} \leq\left\|v_{0}\right\|_{B_{p, r}^{s}} e^{C \int_{0}^{t}\left(\left\|u^{(n)}(\tau)\right\|_{B_{p, r}^{s}}+\left\|v^{(n)}(\tau)\right\|_{B_{p, r}^{s}}\right)^{2} d \tau} \\
&+C \int_{0}^{t} e^{C \int_{\tau}^{t}\left(\left\|u^{(n)}\left(\tau^{\prime}\right)\right\|_{B_{p, r}^{s}},+\left\|v^{(n)}\left(\tau^{\prime}\right)\right\|_{B_{p, r}^{s}}\right)^{2} d \tau^{\prime}} \\
& \times\left\|u^{(n)}(\tau)\right\|_{B_{p, r}^{s}}\left\|v^{(n)}(\tau)\right\|_{B_{p, r}^{s}}^{2} d \tau
\end{aligned}
$$

Combining (24) and (25) we arrive at

$$
\begin{gathered}
\left\|u^{(n+1)}(t)\right\|_{B_{p, r}^{s}}+\left\|v^{(n+1)}(t)\right\|_{B_{p, r}^{s}} \\
\leq\left(\left\|u_{0}\right\|_{B_{p, r}^{s}}+\left\|v_{0}\right\|_{B_{p, r}^{s}}\right) e^{C \int_{0}^{t}\left(\left\|u^{(n)}(\tau)\right\|_{B_{p}^{s}}+\left\|v^{(n)}(\tau)\right\|_{p_{p, r}^{s}}\right)^{2} d \tau} \\
+C \int_{0}^{t} e^{C \int_{\tau}^{t}\left(\left\|u^{(n)}\left(\tau^{\prime}\right)\right\|_{p_{p, r}^{s}}^{s}+\left\|v^{(n)}\left(\tau^{\prime}\right)\right\|_{p_{p, r}^{s}}\right)^{2} d \tau^{\prime}} \\
\times\left(\left\|u^{(n)}(\tau)\right\|_{B_{p, r}^{s}}^{2}\left\|v^{(n)}(\tau)\right\|_{B_{p, r}^{s}}\right. \\
\left.+\left\|u^{(n)}(\tau)\right\|_{B_{p, r}^{s}}\left\|v^{(n)}(\tau)\right\|_{B_{p, r}^{s}}^{2}\right) d \tau \\
\leq\left(\left\|u_{0}\right\|_{B_{p, r}^{s}}+\left\|v_{0}\right\|_{B_{p, r}^{s}}\right) e^{C \int_{0}^{t}\left(\left\|u^{(n)}(\tau)\right\|_{B_{p, r}^{s}}^{s}+\left\|v^{(n)}(\tau)\right\|_{B_{p, r}^{s}}\right)^{2} d \tau} \\
+C \int_{0}^{t} e^{C \int_{\tau}^{t}\left(\left\|u^{(n)}\left(\tau^{\prime}\right)\right\|_{B_{p, r}^{s}, r}+\left\|v^{(n)}\left(\tau^{\prime}\right)\right\|_{p_{p, r}^{s}}\right)^{2} d \tau^{\prime}} \\
\times\left(\left\|u^{(n)}(\tau)\right\|_{B_{p, r}^{s}}+\left\|v^{(n)}(\tau)\right\|_{B_{p, r}^{s}}\right)^{3} d \tau .
\end{gathered}
$$

Choose a $T>0$ such that $4 C\left(\left\|u_{0}\right\|_{B_{p, r}^{s}}+\left\|v_{0}\right\|_{B_{p, r}^{s}}\right)^{2} T<1$ and suppose that for all $t \in[0, T]$,

$$
\begin{aligned}
& \left\|u^{(n)}(t)\right\|_{B_{p, r}^{s}}+\left\|v^{(n)}(t)\right\|_{B_{p, r}^{s}} \\
& \leq \frac{\left\|u_{0}\right\|_{B_{p, r}^{s}}+\left\|v_{0}\right\|_{B_{p, r}^{s}}}{\left[1-4 C\left(\left\|u_{0}\right\|_{B_{p, r}^{s}}+\left\|v_{0}\right\|_{B_{p, r}^{s}}\right)^{2} t\right]^{1 / 2}} .
\end{aligned}
$$


International Journal of Partial Differential Equations

5

Then we have

$$
\begin{aligned}
C \int_{0}^{t}\left(\left\|u^{(n)}(\tau)\right\|_{B_{p, r}^{s}}+\left\|v^{(n)}(\tau)\right\|_{B_{p, r}^{s}}\right)^{2} d \tau \\
\leq C \int_{0}^{t} \frac{\left(\left\|u_{0}\right\|_{B_{p, r}^{s}}+\left\|v_{0}\right\|_{B_{p, r}^{s}}\right)^{2}}{1-4 C\left(\left\|u_{0}\right\|_{B_{p, r}^{s}}+\left\|v_{0}\right\|_{B_{p, r}^{s}}\right)^{2} \tau} d \tau \\
=-\frac{1}{4} \ln \left[1-4 C\left(\left\|u_{0}\right\|_{B_{p, r}^{s}}+\left\|v_{0}\right\|_{B_{p, r}^{s}}\right)^{2} t\right], \\
C \int_{\tau}^{t}\left(\left\|u^{(n)}\left(\tau^{\prime}\right)\right\|_{B_{p, r}^{s}}+\left\|v^{(n)}\left(\tau^{\prime}\right)\right\|_{B_{p, r}^{s}}\right)^{2} d \tau^{\prime} \\
\leq C \int_{\tau}^{t} \frac{\left(\left\|u_{0}\right\|_{B_{p, r}^{s}}+\left\|v_{0}\right\|_{B_{p, r}^{s}}\right)^{2}}{1-4 C\left(\left\|u_{0}\right\|_{B_{p, r}^{s}}+\left\|v_{0}\right\|_{B_{p, r}^{s}}\right)^{2} \tau^{\prime}} d \tau^{\prime} \\
=-\frac{1}{4} \ln \left[1-4 C\left(\left\|u_{0}\right\|_{B_{p, r}^{s}}+\left\|v_{0}\right\|_{B_{p, r}^{s}}\right)^{2} t\right] \\
+\frac{1}{4} \ln \left[1-4 C\left(\left\|u_{0}\right\|_{B_{p, r}^{s}}+\left\|v_{0}\right\|_{B_{p, r}^{s}}\right)^{2} \tau\right],
\end{aligned}
$$

which along with (26) leads to

$$
\begin{aligned}
\left\|u^{(n+1)}(t)\right\|_{B_{p, r}^{s}}+\left\|v^{(n+1)}(t)\right\|_{B_{p, r}^{s}} & \frac{\left\|u_{0}\right\|_{B_{p, r}^{s}}+\left\|v_{0}\right\|_{B_{p, r}^{s}}}{\left[1-4 C\left(\left\|u_{0}\right\|_{B_{p, r}^{s}}+\left\|v_{0}\right\|_{B_{p, r}^{s}}\right)^{2} t\right]^{1 / 4}} \\
& +\frac{C}{\left[1-4 C\left(\left\|u_{0}\right\|_{B_{p, r}^{s}}+\left\|v_{0}\right\|_{B_{p, r}^{s}}\right)^{2} t\right]^{1 / 4}} \\
& \times \int_{0}^{t}\left[1-4 C\left(\left\|u_{0}\right\|_{B_{p, r}^{s}}+\left\|v_{0}\right\|_{B_{p, r}^{s}}\right)^{2} \tau\right]^{1 / 4} \\
& \times \frac{\left(\left\|u_{0}\right\|_{B_{p, r}^{s}}+\left\|v_{0}\right\|_{B_{p, r}^{s}}\right)^{3}}{\left[1-4 C\left(\left\|u_{0}\right\|_{B_{p, r}^{s}}+\left\|v_{0}\right\|_{B_{p, r}^{s}}\right)^{2} \tau\right]^{3 / 2}} d \tau \\
= & \frac{\left\|u_{0}\right\|_{B_{p, r}^{s}}+\left\|v_{0}\right\|_{B_{p, r}^{s}}}{\left[1-4 C\left(\left\|u_{0}\right\|_{B_{p, r}^{s}}+\left\|v_{0}\right\|_{B_{p, r}^{s}}\right)^{2} t\right]^{1 / 4}} \\
& \times\left(1+C \int_{0}^{t} \frac{\left(\left\|u_{0}\right\|_{B_{p, r}^{s}}+\left\|v_{0}\right\|_{B_{p, r}^{s}}\right)^{2}}{\left[1-4 C\left(\left\|u_{0}\right\|_{B_{p, r}^{s}}+\left\|v_{0}\right\|_{B_{p, r}^{s}}\right)^{2} \tau\right]^{5 / 4}} d \tau\right.
\end{aligned}
$$

$$
\begin{aligned}
= & \frac{\left\|u_{0}\right\|_{B_{p, r}^{s}}+\left\|v_{0}\right\|_{B_{p, r}^{s}}}{\left[1-4 C\left(\left\|u_{0}\right\|_{B_{p, r}^{s}}+\left\|v_{0}\right\|_{P_{p, r}^{s}}\right)^{2} t\right]^{1 / 4}} \\
& \times\left(1+\frac{1}{\left[1-4 C\left(\left\|u_{0}\right\|_{B_{p, r}^{s}}+\left\|v_{0}\right\|_{B_{p, r}^{s}}\right)^{2} t\right]^{1 / 4}}-1\right) \\
= & \frac{\left\|u_{0}\right\|_{B_{p, r}^{s}}+\left\|v_{0}\right\|_{B_{p, r}^{s}}^{s}}{\left[1-4 C\left(\left\|u_{0}\right\|_{B_{p, r}^{s}}+\left\|v_{0}\right\|_{B_{p, r}^{s}}\right)^{2} t\right]^{1 / 2}} .
\end{aligned}
$$

Therefore, we conclude that $\left(u^{(n)}, v^{(n)}\right)_{n \in \mathbb{N}} \in \mathscr{C}\left([0, T] ; B_{p, r}^{s}\right)^{2}$. If $s>3+(1 / p)$, then $B_{p, r}^{s-3}$ is an algebra. Hence, according to Proposition 3(7) we have

$$
\begin{aligned}
& \|- a\left(u^{(n)}, v^{(n)}\right) \partial_{x} m^{(n+1)} \|_{B_{p, r}^{s-3}} \\
& \leq C\left\|a\left(u^{(n)}, v^{(n)}\right)\right\|_{B_{p, r}^{s-3}}\left\|\partial_{x} m^{(n+1)}\right\|_{B_{p, r}^{s-3}} \\
& \leq C\left\|u_{x}^{(n)} v_{x}^{(n)}-u^{(n)} v^{(n)}+u^{(n)} v_{x}^{(n)}-u_{x}^{(n)} v^{(n)}\right\|_{B_{p, r}^{s-3}}\left\|m^{(n+1)}\right\|_{B_{p, r}^{s-2}} \\
& \leq C\left\|u^{(n)}\right\|_{B_{p, r}^{s}}\left\|v^{(n)}\right\|_{B_{p, r}^{s}}\left\|u^{(n+1)}\right\|_{B_{p, r}^{s}} \\
&\left\|m^{(n)}\left[a\left(u^{(n)}, v^{(n)}\right)\right]_{x}\right\|_{B_{p, r}^{s-3}} \leq C\left\|m^{(n)}\right\|_{B_{p, r}^{s-3}}\left\|\left[a\left(u^{(n)}, v^{(n)}\right)\right]_{x}\right\|_{B_{p, r}^{s-3}} \\
& \leq C\left\|m^{(n)}\right\|_{B_{p, r}^{s-3}}\left\|u_{x}^{(n)} v_{x}^{(n)}-u^{(n)} v^{(n)}+u^{(n)} v_{x}^{(n)}-u_{x}^{(n)} v^{(n)}\right\|_{B_{p, r}^{s-2}} \\
& \leq C\left\|u^{(n)}\right\|_{B_{p, r}^{s}}\left\|u^{(n)}\right\|_{B_{p, r}^{s}}\left\|v^{(n)}\right\|_{B_{p, r}^{s}} \\
& \leq C\left\|u^{(n)}\right\|_{B_{p, r}^{s}}^{2}\left\|v^{(n)}\right\|_{B_{p, r}^{s}} \cdot
\end{aligned}
$$

If $\max \{2+(1 / p), 5 / 2\}<s \leq 3+(1 / p)$, using the Moser-type estimate (14), we obtain

$$
\begin{aligned}
& \left\|-a\left(u^{(n)}, v^{(n)}\right) \partial_{x} m^{(n+1)}\right\|_{B_{p, r}^{s-3}} \\
& \leq C\left\|a\left(u^{(n)}, v^{(n)}\right)\right\|_{B_{p, r}^{s-2}}\left\|\partial_{x} m^{(n+1)}\right\|_{B_{p, r}^{s-3}} \\
& \leq C\left\|u_{x}^{(n)} v_{x}^{(n)}-u^{(n)} v^{(n)}+u^{(n)} v_{x}^{(n)}-u_{x}^{(n)} v^{(n)}\right\|_{B_{p, r}^{s-2}}\left\|m^{(n+1)}\right\|_{B_{p, r}^{s-2}} \\
& \leq C\left\|u^{(n)}\right\|_{B_{p, r}^{s}}\left\|v^{(n)}\right\|_{B_{p, r}^{s}}\left\|u^{(n+1)}\right\|_{B_{p, r}^{s}},
\end{aligned}
$$




$$
\begin{aligned}
& \left\|m^{(n)}\left[a\left(u^{(n)}, v^{(n)}\right)\right]_{x}\right\|_{B_{p, r}^{s-3}} \\
& \leq C\left\|m^{(n)}\right\|_{B_{p, r}^{s-s}}\left\|\left[a\left(u^{(n)}, v^{(n)}\right)\right]_{x}\right\|_{B_{p, r}^{s-3}} \\
& \leq C\left\|m^{(n)}\right\|_{B_{p, r}^{s-2}}\left\|u_{x}^{(n)} v_{x}^{(n)}-u^{(n)} v^{(n)}+u^{(n)} v_{x}^{(n)}-u_{x}^{(n)} v^{(n)}\right\|_{B_{p, r}^{s-2}} \\
& \leq C\left\|u^{(n)}\right\|_{B_{p, r}^{s}}\left\|u^{(n)}\right\|_{B_{p, r}^{s}}\left\|v^{(n)}\right\|_{B_{p, r}^{s}} \\
& \leq C\left\|u^{(n)}\right\|_{B_{p, r}^{s}}^{2}\left\|v^{(n)}\right\|_{B_{p, r}^{s}} .
\end{aligned}
$$

Thus, using the first equation in (21) and combining (30)-(31) with (29), we have

$$
\begin{aligned}
\left\|\partial_{t} m^{(n+1)}(t)\right\|_{B_{p, r}^{s-3}} \leq & \left\|-a\left(u^{(n)}, v^{(n)}\right) \partial_{x} m^{(n+1)}\right\|_{B_{p, r}^{s},} \\
& +\left\|m^{(n)}\left[a\left(u^{(n)}, v^{(n)}\right)\right]_{x}\right\|_{B_{p, r}^{s-3}} \\
\leq & C\left(\left\|u^{(n)}\right\|_{B_{p, r}^{s}}\left\|v^{(n)}\right\|_{B_{p, r}^{s}}\left\|u^{(n+1)}\right\|_{B_{p, r}^{s}}\right. \\
& \left.+\left\|u^{(n)}\right\|_{B_{p, r}^{s}}^{2}\left\|v^{(n)}\right\|_{B_{p, r}^{s}}\right) \\
\leq & \frac{\left(\left\|u_{0}\right\|_{B_{p, r}^{s}}+\left\|v_{0}\right\|_{B_{p, r}^{s}}\right)^{3}}{\left[1-4 C\left(\left\|u_{0}\right\|_{B_{p, r}^{s}}+\left\|v_{0}\right\|_{B_{p, r}^{s}}\right)^{2} t\right]^{3 / 2}} .
\end{aligned}
$$

In a similar way, we can obtain that

$$
\left\|\partial_{t} h^{(n+1)}(t)\right\|_{B_{p, r}^{s-3}} \leq \frac{C\left(\left\|u_{0}\right\|_{B_{p, r}^{s}}+\left\|v_{0}\right\|_{B_{p, r}^{s}}\right)^{3}}{\left[1-4 C\left(\left\|u_{0}\right\|_{B_{p, r}^{s}}+\left\|v_{0}\right\|_{B_{p, r}^{s}}\right)^{2} t\right]^{3 / 2}} .
$$

Thus, (32) and (33) imply that $\left(\partial_{t} u^{(n+1)}, \partial_{t} v^{(n+1)}\right) \quad \epsilon$ $\mathscr{C}\left([0, T] ; B_{p, r}^{s-1}\right)^{2}$. Therefore

$$
\left(u^{(n)}, v^{(n)}\right)_{n \in \mathbb{N}} \in E_{p, r}^{s}(T)^{2} .
$$

Step 3 (convergence). Now we are going to show that $\left(u^{(n)}\right.$, $\left.v^{(n)}\right)_{n \in \mathbb{N}}$ is a Cauchy sequence in $\mathscr{C}\left([0, T] ; B_{p, r}^{s-1}\right)^{2}$. In fact, according to (21), for all $n, l \in \mathbb{N}$, we obtain that

$$
\begin{aligned}
\left\{\partial_{t}+\right. & {\left.\left[-a\left(u^{(n+l)}, v^{(n+l)}\right)\right] \partial_{x}\right\}\left(m^{(n+l+1)}-m^{(n+1)}\right)(t) } \\
& =g_{1}\left(u^{(n)}, v^{(n)}, u^{(n+l)}, v^{(n+l)}, m^{(n)}, m^{(n+1)}, m^{(n+l)}\right), \\
\left\{\partial_{t}+\right. & {\left.\left[-a\left(u^{(n+l)}, v^{(n+l)}\right)\right] \partial_{x}\right\}\left(h^{(n+l+1)}-h^{(n+1)}\right)(t) } \\
& =g_{2}\left(u^{(n)}, v^{(n)}, u^{(n+l)}, v^{(n+l)}, h^{(n)}, h^{(n+1)}, h^{(n+l)}\right),
\end{aligned}
$$

where

$$
\begin{aligned}
g_{1}\left(u^{(n)}, v^{(n)}, u^{(n+l)}, v^{(n+l)}, m^{(n)}, m^{(n+1)}, m^{(n+l)}\right) \\
=\left(m^{(n+l)}-m^{(n)}\right)\left[a\left(u^{(n)}, v^{(n)}\right)\right]_{x} \\
+\left[a\left(u^{(n+l)}, v^{(n+l)}\right)-a\left(u^{(n)}, v^{(n)}\right)\right] \partial_{x} m^{(n+1)} \\
+\left[a\left(u^{(n+l)}, v^{(n+l)}\right)-a\left(u^{(n)}, v^{(n)}\right)\right]_{x} m^{(n+l)}, \\
g_{2}\left(u^{(n)}, v^{(n)}, u^{(n+l)}, v^{(n+l)}, h^{(n)}, h^{(n+1)}, h^{(n+l)}\right) \\
=\left(h^{(n+l)}-h^{(n)}\right)\left[a\left(u^{(n)}, v^{(n)}\right)\right]_{x} \\
+\left[a\left(u^{(n+l)}, v^{(n+l)}\right)-a\left(u^{(n)}, v^{(n)}\right)\right] \partial_{x} h^{(n+1)} \\
+\left[a\left(u^{(n+l)}, v^{(n+l)}\right)-a\left(u^{(n)}, v^{(n)}\right)\right]_{x} h^{(n+l)} .
\end{aligned}
$$

According to (17), for every $t \in[0, T]$, the following inequality holds:

$$
\begin{gathered}
\left\|m^{(n+l+1)}(t)-m^{(n+1)}(t)\right\|_{B_{p, r}^{s-3}} \\
\leq\left\|m_{0}^{(n+l+1)}-m_{0}^{(n+1)}\right\|_{B_{p, r}^{s-3}} e^{C \int_{0}^{t}\left\|\partial_{x}\left[-a\left(u^{(n+)}, v^{(n+1)}\right)\right](\tau)\right\|_{B_{p, r}^{s-4}} d \tau} \\
+C \int_{0}^{t} e^{C \int_{\tau}^{t}\left\|\partial_{x}\left[-a\left(u^{(n+1)}, v^{(n+l)}\right)\right]\left(\tau^{\prime}\right)\right\|_{B_{p, r}^{s-4}} d \tau^{\prime}} \\
\times \| g_{1}\left(u^{(n)}, v^{(n)}, u^{(n+l)}, v^{(n+l)},\right. \\
\left.m^{(n)}, m^{(n+1)}, m^{(n+l)}\right)(\tau) \|_{B_{p, r}^{s-3}} d \tau,
\end{gathered}
$$

is equivalent to

$$
\begin{gathered}
\left\|u^{(n+l+1)}(t)-u^{(n+1)}(t)\right\|_{B_{p, r}^{s-1}} \\
\leq\left\|u_{0}^{(n+l+1)}-u_{0}^{(n+1)}\right\|_{B_{p, r}^{s-1}} e^{C \int_{0}^{t}\left\|\partial_{x}\left[a\left(u^{(n+1)}, v^{(n+l)}\right)\right](\tau)\right\|_{B_{p, r}^{s-r}} d \tau} \\
+\int_{0}^{t} e^{C \int_{\tau}^{t}\left\|\partial_{x}\left[a\left(u^{(n+1)}, v^{(n+1)}\right)\right]\left(\tau^{\prime}\right)\right\|_{B_{p, r}^{s-4}} d \tau^{\prime}} \\
\times \| g_{1}\left(u^{(n)}, v^{(n)}, u^{(n+l)}, v^{(n+l)},\right. \\
\left.m^{(n)}, m^{(n+1)}, m^{(n+l)}\right)(\tau) \|_{B_{p, r}^{s, 3}} d \tau .
\end{gathered}
$$

If $\max \{2+(1 / p), 5 / 2\}<s \leq 3+(1 / p)$, using the Moser-type estimate (14), we obtain

$$
\begin{aligned}
\left\|\partial_{x}\left[-a\left(u^{(n+l)}, v^{(n+l)}\right)\right]\right\|_{B_{p, r}^{s-4}} \\
\leq C\left\|a\left(u^{(n+l)}, v^{(n+l)}\right)\right\|_{B_{p, r}^{s-3}} \\
=C \| u_{x}^{(n+l)} v_{x}^{(n+l)}-u^{(n+l)} v^{(n+l)} \\
\quad+u^{(n+l)} v_{x}^{(n+l)}-u_{x}^{(n+l)} v^{(n+l)} \|_{B_{p, r}^{s-3}}
\end{aligned}
$$


International Journal of Partial Differential Equations

7

$$
\begin{aligned}
& \leq C\left(\left\|u_{x}^{(n+l)}\right\|_{B_{p, r}^{s-2}}\left\|v_{x}^{(n+l)}\right\|_{B_{p, r}^{s-3}}\right. \\
& \quad+\left\|u^{(n+l)}\right\|_{B_{p, r}^{s-r}}\left\|v^{(n+l)}\right\|_{B_{p, r}^{s-3}}+\left\|u^{(n+l)}\right\|_{B_{p, r}^{s-2}}\left\|v_{x}^{(n+l)}\right\|_{B_{p, r}^{s-3}} \\
& \left.\quad+\left\|u_{x}^{(n+l)}\right\|\left\|_{B_{p, r}^{s-2}}\right\| v^{(n+l)} \|_{B_{p, r}^{s-3}}\right) \\
& \leq C\left\|u^{(n+l)}\right\|_{B_{p, r}^{s}}\left\|v^{(n+l)}\right\|_{B_{p, r}^{s}} \\
& \leq C\left(\left\|u^{(n+l)}\right\|_{B_{p, r}^{s}}+\left\|v^{(n+l)}\right\|_{B_{p, r}^{s}}\right)^{2},
\end{aligned}
$$

$$
\begin{aligned}
& \left\|\left(m^{(n+l)}-m^{(n)}\right)\left[a\left(u^{(n)}, v^{(n)}\right)\right]_{x}\right\|_{B_{p, r}^{s-3}} \\
& \leq C\left\|m^{(n+l)}-m^{(n)}\right\|_{B_{p, r}^{s-s}}\left\|\left[a\left(u^{(n)}, v^{(n)}\right)\right]_{x}\right\|_{B_{p, r}^{s-2}} \\
& \leq C\left\|u^{(n+l)}-u^{(n)}\right\|_{B_{p, r}^{s-1}} \\
& \quad \times\left\|u_{x}^{(n)} v_{x}^{(n)}-u^{(n)} v^{(n)}+u^{(n)} v_{x}^{(n)}-u_{x}^{(n)} v^{(n)}\right\|_{B_{p, r}^{s-1}}
\end{aligned}
$$$$
\leq C\left\|u^{(n+l)}-u^{(n)}\right\|_{B_{p, r}^{s-1}}\left\|u^{(n)}\right\|_{B_{p, r}^{s},} v^{(n)} \|_{\|_{B_{p, r}^{s}}}
$$$$
\leq C\left\|u^{(n+l)}-u^{(n)}\right\|_{B_{p, r}^{s-1}}\left(\left\|u^{(n)}\right\|_{B_{p, r}^{s}}^{2}+\left\|v^{(n)}\right\|_{\|_{p, r}^{s}}^{2}\right),
$$

$$
\left\|\left[a\left(u^{(n+l)}, v^{(n+l)}\right)-a\left(u^{(n)}, v^{(n)}\right)\right] \partial_{x} m^{(n+1)}\right\|_{B_{p, r}^{s-3}}
$$$$
\leq C\left\|a\left(u^{(n+l)}, v^{(n+l)}\right)-a\left(u^{(n)}, v^{(n)}\right)\right\|_{B_{p, r}^{s-2}}\left\|\partial_{x} m^{(n+1)}\right\|_{B_{p, r}^{s-3}}
$$$$
\leq C \|\left(u^{(n+l)}-u^{(n)}\right)_{x} v_{x}^{(n+l)}+\left(v^{(n+l)}-v^{(n)}\right)_{x} u_{x}^{(n)}
$$$$
-\left(u^{(n+l)}-u^{(n)}\right) v^{(n+l)}-\left(v^{(n+l)}-v^{(n)}\right) u^{(n)}
$$$$
+\left(u^{(n+l)}-u^{(n)}\right) v_{x}^{(n+l)}+\left(v^{(n+l)}-v^{(n)}\right)_{x} u^{(n)}
$$$$
-\left(u^{(n+l)}-u^{(n)}\right)_{x} v^{(n+l)}
$$$$
-\left(v^{(n+l)}-v^{(n)}\right) u_{x}^{(n)}\left\|_{B_{p, r}^{s-2}}\right\| m^{(n+1)} \|_{B_{p, r}^{s-2}}
$$

$$
\begin{aligned}
& \leq C\left(\left\|u^{(n+l)}-u^{(n)}\right\|_{B_{p, r}^{s-1}}\left\|v^{(n+l)}\right\|_{B_{p, r}^{s}}\right. \\
& \left.+\left\|v^{(n+l)}-v^{(n)}\right\|_{B_{p, r}^{s-1}}\left\|u^{(n)}\right\|_{B_{p, r}^{s}}\right)\left\|u^{(n+1)}\right\|_{\|_{B_{p, r}^{s}}} \\
& \leq C\left(\left\|u^{(n+l)}-u^{(n)}\right\|_{B_{p, r}^{s-1}}\left(\left\|v^{(n+l)}\right\|_{B_{p, r}^{s}}^{2}+\left\|u^{(n+1)}\right\|_{\|_{B_{p, r}^{s}}^{2}}^{2}\right)\right. \\
& \left.+\left\|v^{(n+l)}-v^{(n)}\right\|_{B_{p, r}^{s-1}}\left(\left\|u^{(n)}\right\|_{B_{p, r}^{s}}^{2}+\left\|u^{(n+1)}\right\|_{\|_{B_{p}^{s}}^{s}}^{2}\right)\right),
\end{aligned}
$$

$$
\begin{aligned}
& \left\|\left[a\left(u^{(n+l)}, v^{(n+l)}\right)-a\left(u^{(n)}, v^{(n)}\right)\right]_{x} m^{(n+l)}\right\|_{B_{p, r}^{s-3}} \\
& \leq C\left\|\left[a\left(u^{(n+l)}, v^{(n+l)}\right)-a\left(u^{(n)}, v^{(n)}\right)\right]_{x}\right\|_{B_{p, r}^{s-r}}\left\|m^{(n+1)}\right\|_{B_{p, r}^{s-2}} \\
& \leq C \|\left(u^{(n+l)}-u^{(n)}\right)_{x} v_{x}^{(n+l)}+\left(v^{(n+l)}-v^{(n)}\right) u_{x}^{(n)} \\
& \quad-\left(u^{(n+l)}-u^{(n)}\right) v^{(n+l)}-\left(v^{(n+l)}-v^{(n)}\right) u^{(n)} \\
& +\left(u^{(n+l)}-u^{(n)}\right) v_{x}^{(n+l)}+\left(v^{(n+l)}-v^{(n)}\right) u_{x}^{(n)} \\
& \quad-\left(u^{(n+l)}-u^{(n)}\right)_{x} v^{(n+l)}-\left(v^{(n+l)}-v^{(n)}\right) u_{x}^{(n)} \|_{B_{p, r}^{s-2}} \\
& \times\left\|m^{(n+l)}\right\|_{B_{p, r}^{s}-2} \\
& \leq C\left(\left\|u^{(n+l)}-u^{(n)}\right\|_{B_{p, r}^{s-1}}\left\|v^{(n+l)}\right\|_{B_{p, r}^{s}}+\left\|v^{(n+l)}-v^{(n)}\right\|_{B_{p, r}^{s-1}}\right. \\
& \times \\
& \left.\quad\left\|u^{(n)}\right\|_{B_{p, r}^{s}}\right)\left\|u^{(n+l)}\right\|_{\|_{p, r}^{s}} \\
& \leq C\left(\left\|u^{(n+l)}-u^{(n)}\right\|_{B_{p, r}^{s-1}}\left(\left\|v^{(n+l)}\right\|_{B_{p, r}^{s}}^{2}+\left\|u^{(n+1)}\right\|_{\|_{B_{p}^{s}, r}^{2}}^{2}\right)\right. \\
& \left.\quad+\left\|v^{(n+l)}-v^{(n)}\right\|_{B_{p, r}^{s-1}}\left(\left\|u^{(n)}\right\|_{B_{p, r}^{s}}^{2}+\left\|u^{(n+l)}\right\|_{\|_{B_{p}^{s}, r}^{s}}^{2}\right)\right) .
\end{aligned}
$$

If $s>3+(1 / p)$, then $B_{p, r}^{s-3}$ is an algebra, and we can verify that (39)-(42) also hold true. Thus, we have

$$
\begin{gathered}
\left\|g_{1}\left(u^{(n)}, v^{(n)}, u^{(n+l)}, v^{(n+l)}, m^{(n)}, m^{(n+1)}, m^{(n+l)}\right)(\tau)\right\|_{B_{p, r}^{s-3}} \\
\leq C\left(\left\|u^{(n+l)}-u^{(n)}\right\|_{B_{p, r}^{s-1}}\right. \\
\times\left(\left\|u^{(n)}\right\|_{B_{p, r}^{s}}^{2}+\left\|v^{(n)}\right\|_{B_{p, r}^{s}}^{2}+\left\|u^{(n+1)}\right\|_{\|_{p, r}^{s}}^{2}\right. \\
\left.+\left\|u^{(n+l)}\right\|_{B_{p, r}^{s}}^{2}+\left\|v^{(n+l)}\right\|_{B_{p, r}^{s}}^{2}\right) \\
+\left\|v^{(n+l)}-v^{(n)}\right\|_{B_{p, r}^{s-1}} \\
\left.\times\left(\left\|u^{(n)}\right\|_{B_{p, r}^{s}}^{2}+\left\|u^{(n+1)}\right\|_{\|_{B_{p, r}^{s}}^{s}}^{2}+\left\|u^{(n+l)}\right\|_{\|_{B_{p, r}^{s}}^{s}}^{2}\right)\right) .
\end{gathered}
$$

Plugging (39) and (43) into (38) we get

$$
\begin{aligned}
& \left\|u^{(n+l+1)}(t)-u^{(n+1)}(t)\right\|_{B_{p, r}^{s-1}} \\
& \leq\left\|u_{0}^{(n+l+1)}-u_{0}^{(n+1)}\right\|_{B_{p, r}^{s s}} C \int_{0}^{t}\left(\left\|u^{(n+1)}(\tau)\right\|_{B_{p, r}^{s}}+\left\|v^{(n+1)}(\tau)\right\|_{B_{p, r}^{s}}\right)^{2} d \tau
\end{aligned}
$$

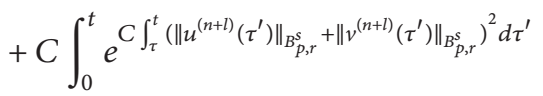

$$
\begin{aligned}
& \times\left(\left\|u^{(n+l)}-u^{(n)}\right\|_{B_{p, r}^{s-1}}\right.
\end{aligned}
$$




$$
\begin{aligned}
& \times\left(\left\|u^{(n)}\right\|_{B_{p, r}^{s}}^{2}+\left\|v^{(n)}\right\|_{B_{p, r}^{s}}^{2}+\left\|u^{(n+1)}\right\|_{\|_{B_{p}^{s} r}}^{2}\right. \\
& \left.+\left\|u^{(n+l)}\right\|_{B_{p, r}^{s}}^{2}+\left\|v^{(n+l)}\right\|_{B_{p, r}^{s}}^{2}\right) \\
& +\left\|v^{(n+l)}-v^{(n)}\right\|_{B_{p, r}^{s-1}} \\
& \left.\times\left(\left\|u^{(n)}\right\|_{B_{p, r}^{s}}^{2}+\left\|u^{(n+1)}\right\|_{\|_{B_{p, r}^{s}}^{2}}^{2} \| u^{(n+l) \|_{\|_{B_{p}, r}}^{2}}\right)\right)(\tau) d \tau .
\end{aligned}
$$

Similarly, we can derive that

$$
\begin{gathered}
\left\|v^{(n+l+1)}(t)-v^{(n+1)}(t)\right\|_{B_{p, r}^{s-1}} \\
\leq\left\|v_{0}^{(n+l+1)}-v_{0}^{(n+1)}\right\|_{B_{p, r}^{s-1}} e^{C \int_{0}^{t}\left(\left\|u^{(n+l)}(\tau)\right\|_{B_{p, r}^{s}}+\left\|v^{(n+l)}(\tau)\right\|_{B_{p, r}^{s}}\right)^{2} d \tau} \\
+C \int_{0}^{t} e^{C \int_{\tau}^{t}\left(\left\|u^{(n+l)}\left(\tau^{\prime}\right)\right\|_{B_{p, r}^{s}}+\left\|v^{(n+l)}\left(\tau^{\prime}\right)\right\|_{B_{p, r}^{s}}\right)^{2} d \tau^{\prime}} \\
\times\left(\left\|u^{(n+l)}-u^{(n)}\right\|_{B_{p, r}^{s-1}}\right. \\
\times\left(\left\|v^{(n+1)}\right\|_{B_{p, r}^{s}}^{2}+\left\|v^{(n+l)}\right\|_{B_{p, r}^{s}}^{2}\right) \\
+\left\|v^{(n+l)}-v^{(n)}\right\|_{B_{p, r}^{s-1}} \\
\times\left(\left\|u^{(n)}\right\|_{B_{p, r}^{s}}^{2}+\left\|v^{(n)}\right\|_{\|_{p, r}^{s}}^{2}\right. \\
\left.\left.+\left\|v^{(n+1)}\right\|_{B_{p, r}^{s}}^{2}+\left\|v^{(n+l)}\right\|_{B_{p, r}^{s}}^{2}\right)\right) d \tau
\end{gathered}
$$

Combining (44) and (45), we have

$$
\begin{aligned}
& \left\|u^{(n+l+1)}(t)-u^{(n+1)}(t)\right\|_{B_{p, r}^{s-1}} \\
& +\left\|v^{(n+l+1)}(t)-v^{(n+1)}(t)\right\|_{B_{p, r}^{s-1}} \\
& \leq\left(\left\|u_{0}^{(n+l+1)}-u_{0}^{(n+1)}\right\|_{B_{p, r}^{s-1}}+\left\|v_{0}^{(n+l+1)}-v_{0}^{(n+1)}\right\|_{B_{p, r}^{s-1}}\right) \\
& \quad \times e^{C \int_{0}^{t}\left(\left\|u^{(n+l)}(\tau)\right\|_{B_{p, r}^{s}}+\left\|v^{(n+l)}(\tau)\right\|_{B_{p, r}^{s}}\right)^{2} d \tau} \\
& +C \int_{0}^{t} e^{C \int_{\tau}^{t}\left(\left\|u^{(n+l)}\left(\tau^{\prime}\right)\right\|_{B_{p, r}^{s}}+\left\|v^{(n+l)}\left(\tau^{\prime}\right)\right\|_{B_{p, r}^{s}}\right)^{2} d \tau^{\prime}} \\
& \quad \times\left(\left\|u^{(n+l)}-u^{(n)}\right\|_{B_{p, r}^{s-1}}+\left\|v^{(n+l)}-v^{(n)}\right\|_{B_{p, r}^{s-1}}\right)
\end{aligned}
$$

$$
\begin{aligned}
\times & \left(\left\|u^{(n)}\right\|_{B_{p, r}^{s}}^{2}+\left\|u^{(n+1)}\right\|_{B_{p, r}^{s}}^{2}+\left\|u^{(n+l)}\right\|_{B_{p, r}^{s}}^{2}\right. \\
& +\left\|v^{(n)}\right\|_{\|_{B_{p, r}^{s}}^{s}}^{2}+\left\|v^{(n+1)}\right\|_{B_{p, r}^{s}}^{2} \\
& \left.+\left\|v^{(n+l)}\right\|_{\|_{B_{p, r}^{s}}}^{2}\right)(\tau) d \tau .
\end{aligned}
$$

Since

$$
\begin{aligned}
\left\|u_{0}^{(n+l+1)}-u_{0}^{(n+1)}\right\|_{B_{p, r}^{s-1}} & =\left\|S_{n+l+1} u_{0}-S_{n+1} u_{0}\right\|_{B_{p, r}^{s-1}} \\
& =\left\|\sum_{q=n+1}^{n+l} \Delta_{q} u_{0}\right\|_{B_{p, r}^{s-1}} \leq C 2^{-n}\left\|u_{0}\right\|_{\|_{B_{p, r}^{s-r}}}, \\
\left\|v_{0}^{(n+l+1)}-v_{0}^{(n+1)}\right\|_{B_{p, r}^{s-1}} & =\left\|S_{n+l+1} u_{0}-S_{n+1} v_{0}\right\|_{B_{p, r}^{s-1}} \\
& =\left\|\sum_{q=n+1}^{n+l} \Delta_{q} v_{0}\right\|_{B_{p, r}^{s-1}} \leq C 2^{-n}\left\|v_{0}\right\|_{\|_{B_{p, r}^{s-1}}},
\end{aligned}
$$

which can be seen on page 2142 of [20], and $\left(u^{(n)}, v^{(n)}\right)_{n \in \mathbb{N}}$ is uniformly bounded in $E_{p, r}^{s}(T)^{2}$, then there exists a constant $C_{T}$ independent of $n$ and $l$ such that, for all $t \in[0, T]$,

$$
\begin{gathered}
\left\|u^{(n+l+1)}(t)-u^{(n+1)}(t)\right\|_{B_{p, r}^{s-1}}+\left\|v^{(n+l+1)}(t)-v^{(n+1)}(t)\right\|_{B_{p, r}^{s-1}} \\
\leq C_{T}\left(2^{-n}+\int_{0}^{t}\left(\left\|\left(u^{(n+l)}-u^{(n)}\right)(\tau)\right\|_{B_{p, r}^{s-1}}\right.\right. \\
\left.\left.+\left\|\left(v^{(n+l)}-v^{(n)}\right)(\tau)\right\|_{B_{p, r}^{s-1}}\right) d \tau\right) .
\end{gathered}
$$

Arguing by induction with respect to the index $n$, we can see that

$$
\begin{aligned}
\left\|u^{(n+l+1)}(t)-u^{(n+1)}(t)\right\|_{L_{T}^{\infty}\left(B_{p, r}^{s-1}\right)}+\left\|v^{(n+l+1)}(t)-v^{(n+1)}(t)\right\|_{L_{T}^{\infty}\left(B_{p, r}^{s-1}\right)} \\
\leq \frac{\left(T C_{T}\right)^{n+1}}{(n+1) !}\left(\left\|u^{(l)}\right\|_{L_{T}^{\infty}\left(B_{p, r}^{s}\right)}+\left\|v^{(l)}\right\|_{L_{T}^{\infty}\left(B_{p, r}^{s}\right)}\right) \\
\quad+C_{T} \sum_{k=0}^{n} 2^{-(n-k)} \frac{\left(T C_{T}\right)^{k}}{k !} .
\end{aligned}
$$

Since $\left\|u^{(l)}\right\|_{L_{T}^{\infty}\left(B_{p, r}^{s}\right)}$ and $\left\|v^{(l)}\right\|_{L_{T}^{\infty}\left(B_{p, r}^{s}\right)}$ are bounded independently of $l$, we conclude that there exists some new constant $C_{T}^{\prime}$ independent of $n$ and $l$ such that

$$
\begin{aligned}
& \left\|u^{(n+l+1)}(t)-u^{(n+1)}(t)\right\|_{L_{T}^{\infty}\left(B_{p, r}^{s-1}\right)}+\left\|v^{(n+l+1)}(t)-v^{(n+1)}(t)\right\|_{L_{T}^{\infty}\left(B_{p, r}^{s-1}\right)} \\
& \leq C_{T}^{\prime} 2^{-n} .
\end{aligned}
$$


Hence $\left(u^{(n)}, v^{(n)}\right)_{n \in \mathbb{N}}$ is a Cauchy sequence in $\mathscr{C}\left([0, T] ; B_{p, r}^{s-1}\right)^{2}$ and converges to some function $(u, v) \in \mathscr{C}\left([0, T] ; B_{p, r}^{s-1}\right)^{2}$.

Step 4 (existence of solution in $\left.E_{p, r}^{s}(T)^{2}\right)$. We will prove that $(u, v)$ belongs to $E_{p, r}^{s}(T)^{2}$ and satisfies (4). Since $\left(u^{(n)}, v^{(n)}\right)$ in uniformly bounded in $L^{\infty}\left([0, T] ; B_{p, r}^{s}\right)^{2}$, the Fatou property for the Besov spaces (Proposition 3(5)) guarantees that $(u, v)$ also belongs to $L^{\infty}\left([0, T) ; B_{p, r}^{s}\right)^{2}$.

On the other hand, as $\left(u^{(n)}, v^{(n)}\right)$ converges to $(u, v)$ in $\mathscr{C}\left([0, T] ; B_{p, r}^{s-1}\right)^{2}$, we conclude that the convergence holds in $\mathscr{C}\left([0, T] ; B_{p, r}^{s^{\prime}}\right)^{2}$ for any $s^{\prime}<s$. In fact, if $s^{\prime} \leq s-1$, then

$$
\begin{aligned}
& \left\|u^{(n)}-u\right\|_{B_{p, r}^{s^{\prime}}}+\left\|v^{(n)}-v\right\|_{B_{p, r}^{s^{\prime}}} \\
& \quad \leq C\left\|u^{(n)}-u\right\|_{B_{p, r}^{s-1}}+C\left\|v^{(n)}-v\right\|_{B_{p, r}^{s-1}} .
\end{aligned}
$$

If $s-1<s^{\prime}<s$, then Proposition 3(6) gives us

$$
\begin{aligned}
\| u^{(n)}- & u\left\|_{B_{p, r}^{s^{\prime}}}+\right\| v^{(n)}-v \|_{B_{p, r}^{s^{\prime}}} \\
\leq & C\left\|u^{(n)}-u\right\|_{B_{p, r}^{s-1}}^{\theta}\left\|u^{(n)}-u\right\|_{B_{p, r}^{s}}^{1-\theta} \\
& +C\left\|v^{(n)}-v\right\|_{B_{p, r}^{s-1}}^{\theta}\left\|v^{(n)}-v\right\|_{B_{p, r}^{s-1}}^{1-\theta},
\end{aligned}
$$

where $\theta=s-s^{\prime}$.

Passing to the limit in (21) and we can easily conclude that $(u, v)$ is indeed a solution to the Cauchy problem (4). Thanks to the fact that $(u, v) \in L^{\infty}\left([0, T] ; B_{p, r}^{s}\right)^{2}$, the right-hand side of the equations

$$
\begin{gathered}
m_{t}-\left(u_{x} v_{x}-u v+u v_{x}-u_{x} v\right) m_{x} \\
=m\left(u_{x} v_{x}-u v+u v_{x}-u_{x} v\right)_{x} \\
h_{t}-\left(u_{x} v_{x}-u v+u v_{x}-u_{x} v\right) h_{x} \\
=h\left(u_{x} v_{x}-u v+u v_{x}-u_{x} v\right)_{x}
\end{gathered}
$$

belongs to $L^{\infty}\left([0, T) ; B_{p, r}^{s-2}\right)^{2}$. In particular, for the case $r<+\infty$, Lemma 5 enables us to conclude that $(u, v) \in$ $\mathscr{C}\left([0, T] ; B_{p, r}^{s^{\prime}}\right)^{2}$ for any $s^{\prime} \leq s$. Finally, using (53) again, we see that $\left(\partial_{t} u, \partial_{t} v\right) \in \mathscr{C}\left([0, T] ; B_{p, r}^{s-1}\right)^{2}$ if $r<+\infty$, and in $\left.L^{\infty}\left([0, T) ; B_{p, r}^{s-1}\right)\right)^{2}$ otherwise. Consequently, $(u, v)$ belongs to $E_{p, r}^{s}(T)^{2}$.

Step 5 (uniqueness of solution). Let $\left(u^{(1)}, v^{(1)}\right)$ and $\left(u^{(2)}, v^{(2)}\right)$ be two given solutions of the Cauchy problem (4) with the initial data $\left(u_{0}^{(1)}, v_{0}^{(1)}\right),\left(u_{0}^{(2)}, v_{0}^{(2)}\right) \in B_{p, r}^{s} \times B_{p, r}^{s}$ satisfying $\left(u^{(1)}, v^{(1)}\right),\left(u^{(2)}, v^{(2)}\right) \in L^{\infty}\left([0, T] ; B_{p, r}^{s}\right) \cap \mathscr{C}\left([0, T] ; \mathcal{S}^{\prime}\right)^{2}$.

Denote $u^{(12)} \triangleq u^{(1)}-u^{(2)}, v^{(12)} \triangleq v^{(1)}-v^{(2)}, m^{(12)} \triangleq$ $m^{(1)}-m^{(2)}$ and $h^{(12)} \triangleq h^{(1)}-h^{(2)}$. It is obvious that $u^{(12)}$, $v^{(12)} \in L^{\infty}\left([0, T] ; B_{p, r}^{s}\right) \cap \mathscr{C}\left([0, T] ; \mathcal{S}^{\prime}\right)$, which implies that $u^{(12)}, v^{(12)} \in \mathscr{C}\left([0, T] ; B_{p, r}^{s-1}\right)$, and $u^{(12)}, v^{(12)}, m^{(12)}$ and $h^{(12)}$ solves the transport equations

$$
\begin{gathered}
\left(m^{(12)}\right) \partial_{t}+\left[-a\left(u^{(1)}, v^{(1)}\right)\right]\left(m^{(12)}\right)_{x} \\
=f_{1}\left(u^{(1)}, u^{(2)}, v^{(1)}, v^{(2)}, m^{(2)}, m^{(12)}\right), \\
\left(h^{(12)}\right) \partial_{t}+\left[-a\left(u^{(1)}, v^{(1)}\right)\right]\left(h^{(12)}\right)_{x} \\
=f_{2}\left(u^{(1)}, u^{(2)}, v^{(1)}, v^{(2)}, h^{(2)}, h^{(12)}\right), \\
\left.m^{(12)}\right|_{t=0}=m_{0}^{(12)} \triangleq m_{0}^{(1)}-m_{0}^{(2)}, \\
\left.h^{(12)}\right|_{t=0}=h_{0}^{(12)} \triangleq h_{0}^{(1)}-h_{0}^{(2)},
\end{gathered}
$$

where

$$
\begin{aligned}
& f_{1}\left(u^{(1)}, u^{(2)}, v^{(1)}, v^{(2)}, m^{(2)}, m^{(12)}\right) \\
&= {\left[\left(a\left(u^{(1)}, v^{(1)}\right)-a\left(u^{(2)}, v^{(2)}\right)\right) m^{(2)}\right]_{x} } \\
&+ a\left(u^{(1)}, v^{(1)}\right) m^{(12)}, \\
& f_{2}\left(u^{(1)}, u^{(2)}, v^{(1)}, v^{(2)}, h^{(2)}, h^{(12)}\right) \\
&=\left[\left(a\left(u^{(1)}, v^{(1)}\right)-a\left(u^{(2)}, v^{(2)}\right)\right) h^{(2)}\right]_{x} \\
&+a\left(u^{(1)}, v^{(1)}\right) h^{(12)} .
\end{aligned}
$$

Applying (17) to the first equation in (54), we obtain that

$$
\begin{aligned}
& \left\|m^{(12)}(t)\right\|_{B_{p, r}^{s-3}} \\
& \leq\left\|m_{0}^{(12)}\right\|_{B_{p, r}^{s-3}} e^{C \int_{0}^{t}\left\|\partial_{x}\left[-a\left(u^{(1)}, v^{(1)}\right)\right](\tau)\right\|_{B_{p, r}^{s-4}} d \tau} \\
& \quad+\int_{0}^{t} e^{C \int_{\tau}^{t}\left\|\partial_{x}\left[-a\left(u^{(1)}, v^{(1)}\right)\right]\left(\tau^{\prime}\right)\right\|_{B_{p, r}^{s-4}} d \tau^{\prime}} \\
& \quad \times\left\|f_{1}\left(u^{(1)}, u^{(2)}, v^{(1)}, v^{(2)}, m^{(2)}, m^{(12)}\right)(\tau)\right\|_{B_{p, r}^{s-3}} d \tau,
\end{aligned}
$$

which is equivalent to

$$
\begin{aligned}
& \left\|u^{(12)}(t)\right\|_{B_{p, r}^{s-1}} \\
& \leq\left\|u_{0}^{(12)}\right\|_{B_{p, r}^{s-1}} e^{C \int_{0}^{t}\left\|\partial_{x}\left[-a\left(u^{(1)}, v^{(1)}\right)\right](\tau)\right\|_{B_{p, r}^{s-4}} d \tau} \\
& \quad+\int_{0}^{t} e^{C \int_{\tau}^{t}\left\|\partial_{x}\left[-a\left(u^{(1)}, v^{(1)}\right)\right]\left(\tau^{\prime}\right)\right\|_{B_{p, r}^{s-4}} d \tau^{\prime}} \\
& \quad \times\left\|f_{1}\left(u^{(1)}, u^{(2)}, v^{(1)}, v^{(2)}, m^{(2)}, m^{(12)}\right)(\tau)\right\|_{B_{p, r}^{s-3}} d \tau .
\end{aligned}
$$


10

International Journal of Partial Differential Equations

Since $B_{p, r}^{s-2}$ is an algebra when $s>\max \{2+(1 / p), 5 / 2\}$, we get

$$
\begin{aligned}
& \left\|\left[\left(a\left(u^{(1)}, v^{(1)}\right)-a\left(u^{(2)}, v^{(2)}\right)\right) m^{(2)}\right]_{x}\right\|_{B_{p, r}^{s-3}} \\
& \leq C\left\|\left(a\left(u^{(1)}, v^{(1)}\right)-a\left(u^{(2)}, v^{(2)}\right)\right) m^{(2)}\right\|_{B_{p, r}^{s-2}} \\
& \leq C \| u_{x}^{(12)} v_{x}^{(1)}+v_{x}^{(12)} u_{x}^{(2)}-u^{(12)} v^{(1)}-v^{(12)} u^{(2)} \\
& \quad+u^{(12)} v_{x}^{(1)}+v_{x}^{(12)} u^{(2)}-u_{x}^{(12)} v^{(1)}-v^{(12)} u_{x}^{(2)}\left\|_{B_{p, r}^{s-2}}\right\| m^{(2)} \|_{B_{p, r}^{s-2}} \\
& \leq C\left(\left\|u^{(12)}\right\|_{B_{p, r}^{s-1}}\left\|v^{(1)}\right\|_{B_{p, r}^{s}}+\left\|v^{(12)}\right\|_{B_{p, r}^{s-1}}\left\|u^{(2)}\right\|_{B_{p, r}^{s}}\right)\left\|u^{(2)}\right\|_{B_{p, r}^{s}} \\
& \leq C\left(\left\|u^{(12)}\right\|_{B_{p, r}^{s s-1}}+\left\|v^{(12)}\right\|_{B_{p, r}^{s-1}}\right) \\
& \quad \times\left(\left\|u^{(1)}\right\|_{B_{p, r}^{s}}^{2}+\left\|u^{(2)}\right\|_{B_{p, r}^{s}}^{2}+\left\|v^{(1)}\right\|_{B_{p, r}^{s}}^{2}+\left\|v^{(2)}\right\|_{B_{p, r}^{s}}^{2}\right) .
\end{aligned}
$$

If $\max \{2+(1 / p), 5 / 2\}<s \leq 3+(1 / p)$, using the Moser-type estimate (14), we have

$$
\begin{aligned}
& \left\|\partial_{x}\left[-a\left(u^{(1)}, v^{(1)}\right)\right]\right\|_{B_{p, r}^{s-4}} \\
& \leq C\left\|a\left(u^{(1)}, v^{(1)}\right)\right\|_{B_{p, r}^{s-3}} \\
& =C\left\|u_{x}^{(1)} v_{x}^{(1)}-u^{(1)} v^{(1)}+u^{(1)} v_{x}^{(1)}-u_{x}^{(1)} v^{(1)}\right\|_{B_{p, r}^{s-3}} \\
& \leq C\left(\left\|u_{x}^{(1)}\right\|_{B_{p, r}^{s-2}}\left\|v_{x}^{(1)}\right\|_{B_{p, r}^{s-3}}+\left\|u^{(1)}\right\|_{B_{p, r}^{s-2}}\left\|v^{(1)}\right\|_{B_{p, r}^{s-3}}\right. \\
& \left.+\left\|u^{(1)}\right\|_{B_{p, r}^{s-2}}\left\|v_{x}^{(1)}\right\|_{B_{p, r}^{s-3}}+\left\|u_{x}^{(1)}\right\|_{B_{p, r}^{s-2}}\left\|v^{(1)}\right\|_{B_{p, r}^{s-3}}\right) \\
& \leq C\left\|u^{(1)}\right\|_{B_{p, r}^{s}}\left\|v^{(1)}\right\|_{B_{p, r}^{s}} \\
& \leq C\left(\left\|u^{(1)}\right\|_{B_{p, r}^{s}}^{2}+\left\|u^{(2)}\right\|_{B_{p, r}^{s}}^{2}+\left\|v^{(1)}\right\|_{B_{p, r}^{s}}^{2}+\left\|v^{(2)}\right\|_{B_{p, r}^{s}}^{2}\right), \\
& \left\|a\left(u^{(1)}, v^{(1)}\right) m^{(12)}\right\|_{B_{p, r}^{s-3}} \\
& \leq C\left\|a\left(u^{(1)}, v^{(1)}\right)\right\|_{B_{p, r}^{s-2}}\left\|m^{(12)}\right\|_{B_{p, r}^{s-3}} \\
& =C\left\|u_{x}^{(1)} v_{x}^{(1)}-u^{(1)} v^{(1)}+u^{(1)} v_{x}^{(1)}-u_{x}^{(1)} v^{(1)}\right\|_{B_{p, r}^{s-2}}\left\|m^{(12)}\right\|_{B_{p, r}^{s-3}} \\
& \leq C\left(\left\|u_{x}^{(1)}\right\|_{B_{p, r}^{s-2}}\left\|v_{x}^{(1)}\right\|_{B_{p, r}^{s-2}}+\left\|u^{(1)}\right\|_{B_{p, r}^{s-2}}\left\|v^{(1)}\right\|_{B_{p, r}^{s-2}}\right. \\
& \left.+\left\|u^{(1)}\right\|_{B_{p, r}^{s-2}}\left\|v_{x}^{(1)}\right\|_{B_{p, r}^{s-2}}+\left\|u_{x}^{(1)}\right\|_{B_{p, r}^{s-2}}\left\|v^{(1)}\right\|_{B_{p, r}^{s-2}}\right)\left\|u^{(12)}\right\|_{B_{p, r}^{s-1}} \\
& \leq C\left\|u^{(1)}\right\|_{B_{p, r}^{s}}\left\|v^{(1)}\right\|_{B_{p, r}^{s}}\left\|u^{(12)}\right\|_{B_{p, r}^{s-1}} \\
& \leq C\left(\left\|u^{(12)}\right\|_{B_{p, r}^{s-1}}+\left\|v^{(12)}\right\|_{B_{p, r}^{s-1}}\right) \\
& \times\left(\left\|u^{(1)}\right\|_{B_{p, r}^{s}}^{2}+\left\|u^{(2)}\right\|_{B_{p, r}^{s}}^{2}+\left\|v^{(1)}\right\|_{B_{p, r}^{s}}^{2}+\left\|v^{(2)}\right\|_{B_{p, r}^{s}}^{2}\right) .
\end{aligned}
$$

(60)
If $s>3+(1 / p)$, then $B_{p, r}^{s-3}$ is an algebra. We directly obtain that

$$
\begin{aligned}
& \left\|\partial_{x}\left[-a\left(u^{(1)}, v^{(1)}\right)\right]\right\|_{B_{p, r}^{s-4}} \\
& \leq C\left\|a\left(u^{(1)}, v^{(1)}\right)\right\|_{B_{p, r}^{s-3}} \\
& =C\left\|u_{x}^{(1)} v_{x}^{(1)}-u^{(1)} v^{(1)}+u^{(1)} v_{x}^{(1)}-u_{x}^{(1)} v^{(1)}\right\|_{B_{p, r}^{s-3}} \\
& \leq C\left(\left\|u_{x}^{(1)}\right\|_{B_{p, r}^{s-3}}\left\|v_{x}^{(1)}\right\|_{B_{p, r}^{s-3}}+\left\|u^{(1)}\right\|_{B_{p, r}^{s-3}}\left\|v^{(1)}\right\|_{B_{p, r}^{s-3}}\right. \\
& \left.\quad+\left\|u^{(1)}\right\|_{B_{p, r}^{s-3}}\left\|v_{x}^{(1)}\right\|_{B_{p, r}^{s-3}}+\left\|u_{x}^{(1)}\right\|_{B_{p, r}^{s-3}}\left\|v^{(1)}\right\|_{B_{p, r}^{s-3}}\right) \\
& \leq C\left\|u^{(1)}\right\|_{B_{p, r}^{s}}\left\|v^{(1)}\right\|_{B_{p, r}^{s}} \\
& \leq C\left(\left\|u^{(1)}\right\|_{B_{p, r}^{s}}^{2}+\left\|u^{(2)}\right\|_{B_{p, r}^{s}}^{2}+\left\|v^{(1)}\right\|_{B_{p, r}^{s}}^{2}+\left\|v^{(2)}\right\|_{B_{p, r}^{s}}^{2}\right)
\end{aligned}
$$$$
\left\|a\left(u^{(1)}, v^{(1)}\right) m^{(12)}\right\|_{B_{p, r}^{s-3}}
$$$$
\leq C\left\|a\left(u^{(1)}, v^{(1)}\right)\right\|_{B_{p, r}^{s-3}}\left\|m^{(12)}\right\|_{B_{p, r}^{s-3}}
$$$$
=C\left\|u_{x}^{(1)} v_{x}^{(1)}-u^{(1)} v^{(1)}+u^{(1)} v_{x}^{(1)}-u_{x}^{(1)} v^{(1)}\right\|_{B_{p, r}^{s-3}}\left\|m^{(12)}\right\|_{B_{p, r}^{s-3}}
$$$$
\leq C\left(\left\|u_{x}^{(1)}\right\|_{B_{p, r}^{s-3}}\left\|v_{x}^{(1)}\right\|_{B_{p, r}^{s-3}}+\left\|u^{(1)}\right\|_{B_{p, r}^{s-3}}\left\|v^{(1)}\right\|_{B_{p, r}^{s-3}}\right.
$$$$
\left.+\left\|u^{(1)}\right\|_{B_{p, r}^{s-3}}\left\|v_{x}^{(1)}\right\|_{B_{p, r}^{s-3}}+\left\|u_{x}^{(1)}\right\|_{B_{p, r}^{s-3}}\left\|v^{(1)}\right\|_{B_{p, r}^{s-3}}\right)\left\|u^{(12)}\right\|_{B_{p, r}^{s-1}}
$$$$
\leq C\left\|u^{(1)}\right\|_{B_{p, r}^{s}}\left\|v^{(1)}\right\|_{B_{p, r}^{s}}\left\|u^{(12)}\right\|_{B_{p, r}^{s-1}}
$$$$
\leq C\left(\left\|u^{(12)}\right\|_{B_{p, r}^{s-1}}+\left\|v^{(12)}\right\|_{B_{p, r}^{s-1}}\right)
$$$$
\times\left(\left\|u^{(1)}\right\|_{B_{p, r}^{s}}^{2}+\left\|u^{(2)}\right\|_{B_{p, r}^{s}}^{2}+\left\|v^{(1)}\right\|_{B_{p, r}^{s}}^{2}+\left\|v^{(2)}\right\|_{B_{p, r}^{s}}^{2}\right) .
$$

It follows from (58), (60), and (62) that

$$
\begin{aligned}
\left\|f_{1}\left(u^{(1)}, u^{(2)}, v^{(1)}, v^{(2)}, m^{(2)}, m^{(12)}\right)\right\|_{B_{p, r}^{s-3}} \\
\leq C\left(\left\|u^{(12)}\right\|_{B_{p, r}^{s-1}}+\left\|v^{(12)}\right\|_{B_{p, r}^{s-1}}\right) \\
\quad \times\left(\left\|u^{(1)}\right\|_{B_{p, r}^{s}}^{2}+\left\|u^{(2)}\right\|_{B_{p, r}^{s}}^{2}+\left\|v^{(1)}\right\|_{B_{p, r}^{s}}^{2}+\left\|v^{(2)}\right\|_{B_{p, r}^{s}}^{2}\right) .
\end{aligned}
$$


International Journal of Partial Differential Equations

11

Inserting (63) and (59) (or (61)) into (57), we find that

$$
\begin{aligned}
& \left\|u^{(12)}(t)\right\|_{B_{p, r}^{s-1}}
\end{aligned}
$$

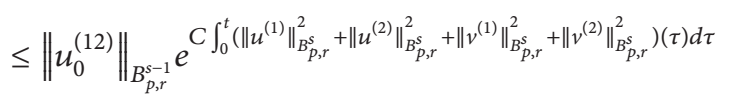

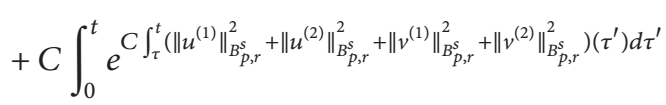

$$
\begin{aligned}
& \times\left(\left\|u^{(12)}\right\|_{B_{p, r}^{s-1}}+\left\|v^{(12)}\right\|_{B_{p, r}^{s-1}}\right) \\
& \times\left(\left\|u^{(1)}\right\|_{B_{p, r}^{s}}^{2}+\left\|u^{(2)}\right\|_{B_{p, r}^{s}}^{2}\right. \\
& \left.+\left\|v^{(1)}\right\|_{B_{p, r}^{s}}^{2}+\left\|v^{(2)}\right\|_{B_{p, r}^{s}}^{2}\right) d \tau \text {. }
\end{aligned}
$$

For the component $v$ in (54), we derive the similar result as follows:

$$
\begin{aligned}
& \left\|v^{(12)}(t)\right\|_{B_{p, r}^{s-1}}
\end{aligned}
$$

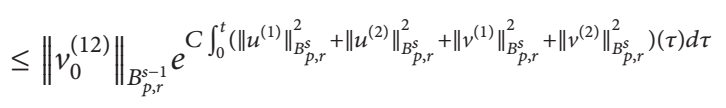

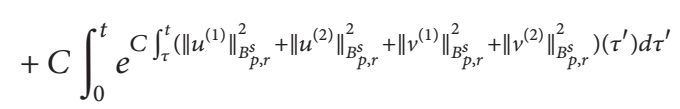

$$
\begin{aligned}
& \times\left(\left\|u^{(12)}\right\|_{B_{p, r}^{s-1}}+\left\|v^{(12)}\right\|_{B_{p, r}^{s-1}}\right) \\
& \times\left(\left\|u^{(1)}\right\|_{B_{p, r}^{s}}^{2}+\left\|u^{(2)}\right\|_{p_{p, r}^{s}}^{2}\right. \\
& \left.+\left\|v^{(1)}\right\|_{B_{p, r}^{s}}^{2}+\left\|v^{(2)}\right\|_{B_{p, r}^{s}}^{2}\right)(\tau) d \tau \text {. }
\end{aligned}
$$

Then, it follows from (64) and (65) that

$$
\begin{gathered}
\left\|u^{(12)}(t)\right\|_{B_{p, r}^{s-1}}+\left\|v^{(12)}(t)\right\|_{B_{p, r}^{s-1}} \\
\leq\left(\left\|u_{0}^{(12)}\right\|_{B_{p, r}^{s-1}}+\left\|v_{0}^{(12)}\right\|_{B_{p, r}^{s-1}}\right) \\
\times e^{C \int_{0}^{t}\left(\left\|u^{(1)}\right\|_{B_{p, r}^{s}}^{2}+\left\|u^{(2)}\right\|_{p_{p, r}^{s}}^{2}+\left\|v^{(1)}\right\|_{B_{p, r}^{s}}^{2}+\left\|v^{(2)}\right\|_{B_{p, r}^{s}}^{2}\right)(\tau) d \tau} \\
+C \int_{0}^{t} e^{C \int_{\tau}^{t}\left(\left\|u^{(1)}\right\|_{B_{p, r}^{s}}^{2}+\left\|u^{(2)}\right\|_{B_{p, r}^{s}}^{2}+\left\|v^{(1)}\right\|_{B_{p, r}^{s}}^{2}+\left\|v^{(2)}\right\|_{B_{p, r}^{s}}^{2}\right)\left(\tau^{\prime}\right) d \tau^{\prime}} \\
\times\left(\left\|u^{(12)}\right\|_{B_{p, r}^{s-1}}+\left\|v^{(12)}\right\|_{B_{p, r}^{s-1}}\right) \\
\times\left(\left\|u^{(1)}\right\|_{B_{p, r}^{s}}^{2}+\left\|u^{(2)}\right\|_{B_{p, r}^{s}}^{2}\right. \\
\left.+\left\|v^{(1)}\right\|_{B_{p, r}^{s}}^{2}+\left\|v^{(2)}\right\|_{B_{p, r}^{s}}^{2}\right)(\tau) d \tau .
\end{gathered}
$$

Applying Gronwall's lemma, we end up with

$$
\begin{aligned}
& \left\|u^{(12)}(t)\right\|_{B_{p, r}^{s-1}}+\left\|v^{(12)}(t)\right\|_{B_{p, r}^{s-1}} \\
& \leq\left(\left\|u_{0}^{(12)}\right\|_{B_{p, r}^{s-1}}+\left\|v_{0}^{(12)}\right\|_{B_{p, r}^{s-1}}\right) \\
& \quad \times \exp \left\{C \int _ { 0 } ^ { t } \left(\left\|u^{(1)}\right\|_{B_{p, r}^{s}}^{2}+\left\|u^{(2)}\right\|_{B_{p, r}^{s}}^{2}\right.\right. \\
& \left.\left.+\left\|v^{(1)}\right\|_{B_{p, r}^{s}}^{2}+\left\|v^{(2)}\right\|_{B_{p, r}^{s}}^{2}\right)(\tau) d \tau\right\} .
\end{aligned}
$$

Step 6 (continuity with respect to the initial data in $E_{p, r}^{s^{\prime}}(T)^{2}$ with $s^{\prime}<s$ ). When $s^{\prime}=s-1$, the continuity with respect to the initial data can be obtained from Step 5 .

When $s^{\prime}<s-1$, by using Proposition 3(6) and (67) we have

$$
\begin{aligned}
& \left\|u^{(1)}(t)-u^{(2)}(t)\right\|_{B_{p, r}^{s^{\prime}}}+\left\|v^{(1)}(t)-v^{(2)}(t)\right\|_{B_{p, r}^{s^{\prime}}} \\
& \leq C\left\|u^{(1)}(t)-u^{(2)}(t)\right\|_{B_{p, r}^{s_{1}}}^{\theta_{1}}\left\|u^{(1)}(t)-u^{(2)}(t)\right\|_{B_{p, r}^{s-1}}^{1-\theta_{1}} \\
& +C\left\|v^{(1)}(t)-v^{(2)}(t)\right\|_{B_{p, r}^{s_{1}}}^{\theta_{1}}\left\|v^{(1)}(t)-v^{(2)}(t)\right\|_{B_{p, r}^{s-1}}^{1-\theta_{1}} \\
& \leq C\left(\left\|u^{(1)}(t)\right\|_{B_{p, r}^{s_{1}}}+\left\|u^{(2)}(t)\right\|_{B_{p, r}^{s_{1}}}\right)^{\theta_{1}} \\
& \times\left(\left\|u_{0}^{(1)}-u_{0}^{(2)}\right\|_{B_{p, r}^{s-1}}+\left\|v_{0}^{(1)}-v_{0}^{(2)}\right\|_{B_{p, r}^{s-1}}\right)^{1-\theta_{1}} \\
& \times \exp \left\{C ( 1 - \theta _ { 1 } ) \int _ { 0 } ^ { t } \left(\left\|u^{(1)}\right\|_{B_{p, r}^{s}}^{2}+\left\|u^{(2)}\right\|_{B_{p, r}^{s}}^{2}\right.\right. \\
& \left.\left.+\left\|v^{(1)}\right\|_{B_{p, r}^{s}}^{2}+\left\|v^{(2)}\right\|_{B_{p, r}^{s}}^{2}\right)(\tau) d \tau\right\} \\
& +C\left(\left\|v^{(1)}(t)\right\|_{B_{p, r}^{s_{1}}}+\left\|v^{(2)}(t)\right\|_{B_{p, r}^{s_{1}}}\right)^{\theta_{1}} \\
& \times\left(\left\|u_{0}^{(1)}-u_{0}^{(2)}\right\|_{B_{p, r}^{s-1}}+\left\|v_{0}^{(1)}-v_{0}^{(2)}\right\|_{B_{p, r}^{s-1}}\right)^{1-\theta_{1}} \\
& \times \exp \left\{C ( 1 - \theta _ { 1 } ) \int _ { 0 } ^ { t } \left(\left\|u^{(1)}\right\|_{B_{p, r}^{s}}^{2}+\left\|u^{(2)}\right\|_{B_{p, r}^{s}}^{2}\right.\right. \\
& \left.\left.+\left\|v^{(1)}\right\|_{B_{p, r}^{s}}^{2}+\left\|v^{(2)}\right\|_{B_{p, r}^{s}}^{2}\right)(\tau) d \tau\right\} \\
& \leq C\left[\left(\left\|u^{(1)}(t)\right\|_{B_{p, r}^{s}}+\left\|u^{(2)}(t)\right\|_{B_{p, r}^{s}}\right)^{\theta_{1}}\right. \\
& \left.+\left(\left\|v^{(1)}(t)\right\|_{B_{p, r}^{s}}+\left\|v^{(2)}(t)\right\|_{B_{p, r}^{s}}\right)^{\theta_{1}}\right] \\
& \times\left(\left\|u_{0}^{(1)}-u_{0}^{(2)}\right\|_{B_{p, r}^{s^{\prime}}}^{\theta_{2}}\left\|u_{0}^{(1)}-u_{0}^{(2)}\right\|_{B_{p, r}^{s}}^{1-\theta_{2}}\right. \\
& \left.+\left\|v_{0}^{(1)}-v_{0}^{(2)}\right\|_{B_{p, r}^{s^{\prime}}}^{\theta_{2}}\left\|v_{0}^{(1)}-v_{0}^{(2)}\right\|_{B_{p, r}^{s}}^{1-\theta_{2}}\right)^{1-\theta_{1}}
\end{aligned}
$$




$$
\begin{aligned}
& \times \exp \left\{C ( 1 - \theta _ { 1 } ) \int _ { 0 } ^ { t } \left(\left\|u^{(1)}\right\|_{B_{p, r}^{s}}^{2}+\left\|u^{(2)}\right\|_{B_{p, r}^{s}}^{2}\right.\right. \\
& \left.\left.+\left\|v^{(1)}\right\|_{B_{p, r}^{s}}^{2}+\left\|v^{(2)}\right\|_{B_{p, r}^{s}}^{2}\right)(\tau) d \tau\right\} \\
& \leq C\left[\left(\left\|u^{(1)}(t)\right\|_{B_{p, r}^{s}}+\left\|u^{(2)}(t)\right\|_{B_{p, r}^{s}}\right)^{\theta_{1}}\right. \\
& \left.+\left(\left\|v^{(1)}(t)\right\|_{B_{p, r}^{s}}+\left\|v^{(2)}(t)\right\|_{B_{p, r}^{s}}\right)^{\theta_{1}}\right] \\
& \times\left[\left\|u_{0}^{(1)}-u_{0}^{(2)}\right\|_{B_{p, r}^{s^{\prime}}}^{\theta_{2}}\left(\left\|u_{0}^{(1)}\right\|_{B_{p, r}^{s}}^{1-\theta_{2}}+\left\|u_{0}^{(2)}\right\|_{B_{p, r}^{s}}^{1-\theta_{2}}\right)\right. \\
& \left.+\left\|v_{0}^{(1)}-v_{0}^{(2)}\right\|_{B_{p, r}^{s^{\prime}}}^{\theta_{2}}\left(\left\|v_{0}^{(1)}\right\|_{B_{p, r}^{s}}^{1-\theta_{2}}+\left\|v_{0}^{(2)}\right\|_{B_{p, r}^{s}}^{1-\theta_{2}}\right)\right]^{1-\theta_{1}} \\
& \times \exp \left\{C ( 1 - \theta _ { 1 } ) \int _ { 0 } ^ { t } \left(\left\|u^{(1)}\right\|_{B_{p, r}^{s}}^{2}+\left\|u^{(2)}\right\|_{B_{p, r}^{s}}^{2}\right.\right. \\
& \left.\left.+\left\|v^{(1)}\right\|_{B_{p, r}^{s}}^{2}+\left\|v^{(2)}\right\|_{B_{p, r}^{s}}^{2}\right)(\tau) d \tau\right\}
\end{aligned}
$$

where $s_{1}<s^{\prime}, s^{\prime}=\theta_{1} s_{1}+\left(1-\theta_{1}\right)(s-1)$ and $s-1=\theta_{2} s^{\prime}+\left(1-\theta_{2}\right) s$.

When $s-1<s^{\prime}<s$, by using Proposition 3(6) and (67) again, we have

$$
\begin{aligned}
& \left\|u^{(1)}(t)-u^{(2)}(t)\right\|_{B_{p, r}^{s^{\prime}}}+\left\|v^{(1)}(t)-v^{(2)}(t)\right\|_{B_{p, r}^{s^{\prime}}} \\
& \leq C\left\|u^{(1)}(t)-u^{(2)}(t)\right\|_{B_{p, r}^{s-1}}^{\theta_{3}}\left\|u^{(1)}(t)-u^{(2)}(t)\right\|_{B_{p, r}^{s}}^{1-\theta_{3}} \\
& +C\left\|v^{(1)}(t)-v^{(2)}(t)\right\|_{B_{p, r}^{s-1}}^{\theta_{1}}\left\|v^{(1)}(t)-v^{(2)}(t)\right\|_{B_{p, r}^{s}}^{1-\theta_{3}} \\
& \leq C\left(\left\|u^{(1)}(t)\right\|_{B_{p, r}^{s}}+\left\|u^{(2)}(t)\right\|_{B_{p, r}^{s}}\right)^{1-\theta_{3}} \\
& \times\left(\left\|u_{0}^{(1)}-u_{0}^{(2)}\right\|_{B_{p, r}^{s-1}}+\left\|v_{0}^{(1)}-v_{0}^{(2)}\right\|_{B_{p, r}^{s-1}}\right)^{\theta_{3}} \\
& \times \exp \left\{C \theta _ { 3 } \int _ { 0 } ^ { t } \left(\left\|u^{(1)}\right\|_{B_{p, r}^{s}}^{2}+\left\|u^{(2)}\right\|_{B_{p, r}^{s}}^{2}\right.\right. \\
& \left.\left.+\left\|v^{(1)}\right\|_{B_{p, r}^{s}}^{2}+\left\|v^{(2)}\right\|_{B_{p, r}^{s}}^{2}\right)(\tau) d \tau\right\} \\
& +C\left(\left\|v^{(1)}(t)\right\|_{B_{p, r}^{s}}+\left\|v^{(2)}(t)\right\|_{B_{p, r}^{s}}\right)^{1-\theta_{3}} \\
& \times\left(\left\|u_{0}^{(1)}-u_{0}^{(2)}\right\|_{B_{p, r}^{s-1}}+\left\|v_{0}^{(1)}-v_{0}^{(2)}\right\|_{B_{p, r}^{s-1}}\right)^{\theta_{3}} \\
& \times \exp \left\{C \theta _ { 3 } \int _ { 0 } ^ { t } \left(\left\|u^{(1)}\right\|_{B_{p, r}^{s}}^{2}+\left\|u^{(2)}\right\|_{B_{p, r}^{s}}^{2}\right.\right. \\
& \left.\left.+\left\|v^{(1)}\right\|_{B_{p, r}^{s}}^{2}+\left\|v^{(2)}\right\|_{B_{p, r}^{s}}^{2}\right)(\tau) d \tau\right\}
\end{aligned}
$$

$$
\begin{aligned}
\leq & C\left[\left(\left\|u^{(1)}(t)\right\|_{B_{p, r}^{s}}+\left\|u^{(2)}(t)\right\|_{B_{p, r}^{s}}\right)^{1-\theta_{3}}\right. \\
& \left.+\left(\left\|v^{(1)}(t)\right\|_{B_{p, r}^{s}}+\left\|v^{(2)}(t)\right\|_{B_{p, r}^{s}}\right)^{1-\theta_{3}}\right] \\
\times & \left(\left\|u_{0}^{(1)}-u_{0}^{(2)}\right\|_{B_{p, r}^{s^{\prime}}}+\left\|v_{0}^{(1)}-v_{0}^{(2)}\right\|_{B_{p, r}^{s^{\prime}}}\right)^{\theta_{3}} \\
\times & \exp \left\{C \theta _ { 3 } \int _ { 0 } ^ { t } \left(\left\|u^{(1)}\right\|_{B_{p, r}^{s}}^{2}+\left\|u^{(2)}\right\|_{B_{p, r}^{s}}^{2}\right.\right. \\
& \left.\left.+\left\|v^{(1)}\right\|_{B_{p, r}^{s}}^{2}+\left\|v^{(2)}\right\|_{B_{p, r}^{s}}^{2}\right)(\tau) d \tau\right\},
\end{aligned}
$$

where $s^{\prime}=\theta_{3}(s-1)+\left(1-\theta_{3}\right) s$.

Step 7 (continuity with respect to the initial data in $E_{p, r}^{s}(T)^{2}$ ). A standard use of a sequence of viscosity approximate solutions $\left(u_{\varepsilon}, v_{\varepsilon}\right)_{\varepsilon}>0$ for the Cauchy problem (4) which converges uniformly in $E_{p, r}^{s}(T)^{2}$ gives the continuity of the solution $(u, v)$ in $E_{p, r}^{s}(T)^{2}$.

Consequently, we complete the proof of the theorem.

When $p=r=2$, the Besov space $B_{p, r}^{s}(\mathbb{R})$ coincides with the Sobolev space $H^{s}(\mathbb{R})$. So we have the following corollary.

Corollary 7. Suppose that $s>5 / 2$ and $\left(u_{0}, v_{0}\right) \in H^{s} \times H^{s}$. Then there exists a time $T>0$ such that the Cauchy problem (4) has a unique solution $(u, v) \in H^{s} \times H^{s}$ and the map $\left(u_{0}, v_{0}\right) \rightarrow$ $(u, v)$ is continuous from a neighborhood of $\left(u_{0}, v_{0}\right)$ in $B_{p, r}^{s} \times B_{p, r}^{s}$ into

$$
\left(\mathscr{C}\left([0, T] ; H^{s}\right) \bigcap \mathscr{C}^{1}\left([0, T] ; H^{s-1}\right)\right)^{2}
$$

\section{Open Problems}

In this paper, we use some standard a priori estimates for linear transport equation to prove the local well-posedness of the Cauchy problem for a modified two-component Camassa-Holm system with cubic nonlinearity in Besov spaces with minimal regularity assumptions on the initial data.

There are some interesting but challenging problems which are still open. The first is whether the index $s=\max \{2+$ $(1 / p), 5 / 2\}$ is sharp for local well-posedness of the Cauchy problem (4). The second is whether the solution can exist globally in time under certain assumptions on the initial data. The third is whether the solution can blow up in finite time with special initial profiles. We will work on these problems in the near future.

\section{Acknowledgments}

This work was supported by the National Nature Science Foundation of China (nos. 11171135, 10420130638), the Nature Science Foundation of Jiangsu (no. BK 2010329), the Natural 
Science Foundation of the Jiangsu Higher Education Institutions of China (no. 09KJB110003), and the Taizhou Social Development Project (no. 2011213).

\section{References}

[1] B. Fuchssteiner, "Some tricks from the symmetry-toolbox for nonlinear equations: generalizations of the Camassa-Holm equation," Physica D, vol. 95, no. 3-4, pp. 229-243, 1996.

[2] P. J. Olver and P. Rosenau, "Tri-Hamiltonian duality between solitons and solitary-wave solutions having compact support," Physical Review E, vol. 53, no. 2, pp. 1900-1906, 1996.

[3] Z. Qiao, "A new integrable equation with cuspons and $W / M-$ shape-peaks solitons," Journal of Mathematical Physics, vol. 47, Article ID 112701, 2006.

[4] Z. Qiao and X. Li, "An integrable equation with nonsmooth solitons," Theoretical and Mathematical Physics, vol. 167, no. 2, pp. 584-589, 2011.

[5] Y. Fu, G. Gui, Y. Liu, and C. Qu, "On the cauchy problem for the integrable Camassa-Holm type equation with cubic nonlinearity," http://arxiv.org/abs/1108.5368.

[6] G. Gui, Y. Liu, P. J. Olver, and C. Qu, "Wave-breaking and peakons for a modified Camassa-Holm equation," Communications in Mathematical Physics, vol. 319, pp. 731-759, 2013.

[7] J. Song, C. Qu, and Z. Qiao, "A new integrable two-component system with cubic nonlinearity," Journal of Mathematical Physics, vol. 52, no. 1, Article ID 013503, 2011.

[8] G. Rodríguez-Blanco, "On the Cauchy problem for the Camassa-Holm equation," Nonlinear Analysis: Theory, Methods and Applications, vol. 46, no. 3, pp. 309-327, 2001.

[9] Z. Yin, "On the cauchy problem for an integrable equation with peakon solutions," Illinois Journal of Mathematics, vol. 47, no. 3 , pp. 649-666, 2003.

[10] L. Ni and Y. Zhou, "Well-posedness and persistence properties for the Novikov equation," Journal of Differential Equations, vol. 250, no. 7, pp. 3002-3021, 2011.

[11] M. Vishik, "Hydrodynamics in Besov spaces," Archive for Rational Mechanics and Analysis, vol. 145, no. 3, pp. 197-214, 1998.

[12] R. Danchin, "A few remarks on the Camassa-Holm equation," Differential and Integral Equations, vol. 14, pp. 953-988, 2001.

[13] R. Danchin, "A note on well-posedness for Camassa-Holm equation," Journal of Differential Equations, vol. 192, no. 2, pp. 429-444, 2003.

[14] R. Danchin and F. Fanelli, "The well-posedness issue for the density-dependent Euler equations in endpoint Besov spaces," Journal des Mathematiques Pures et Appliquees, vol. 96, no. 3, pp. 253-278, 2011.

[15] R. Danchin and P. B. Mucha, "A critical functional framework for the inhomogeneous Navier-Stokes equations in the halfspace," Journal of Functional Analysis, vol. 256, no. 3, pp. 881927, 2009.

[16] R. Danchin, "On the well-posedness of the incompressible density-dependent Euler equations in the $\mathrm{L}_{p}$ framework," Journal of Differential Equations, vol. 248, no. 8, pp. 2130-2170, 2010.

[17] G. Gui and Y. Liu, "On the Cauchy problem for the twocomponent Camassa-Holm system," Mathematische Zeitschrift, vol. 268, no. 1-2, pp. 45-66, 2011.

[18] W. Yan, Y. Li, and Y. Zhang, "The Cauchy problem for the integrable Novikov equation," Journal of Differential Equations, vol. 253, no. 1, pp. 298-318, 2012.
[19] L. Tian, W. Yan, and G. Gui, "On the local well posedness and blow-up solution of a coupled Camassa-Holm equations in Besov Spaces," Journal of Mathematical Physics, vol. 53, no. 1, Article ID 013701, 2012.

[20] K. Yan and Z. Yin, "On the Cauchy problem for a twocomponent Degasperis-Procesi system," Journal of Differential Equations, vol. 252, no. 3, pp. 2131-2159, 2012. 


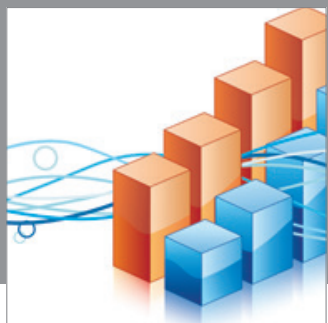

Advances in

Operations Research

mansans

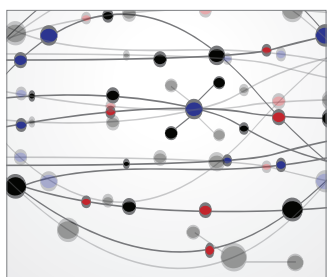

The Scientific World Journal
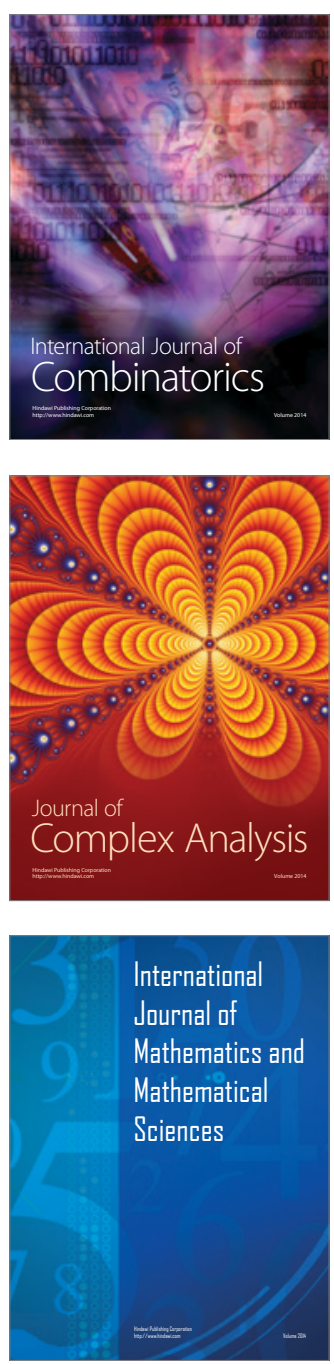
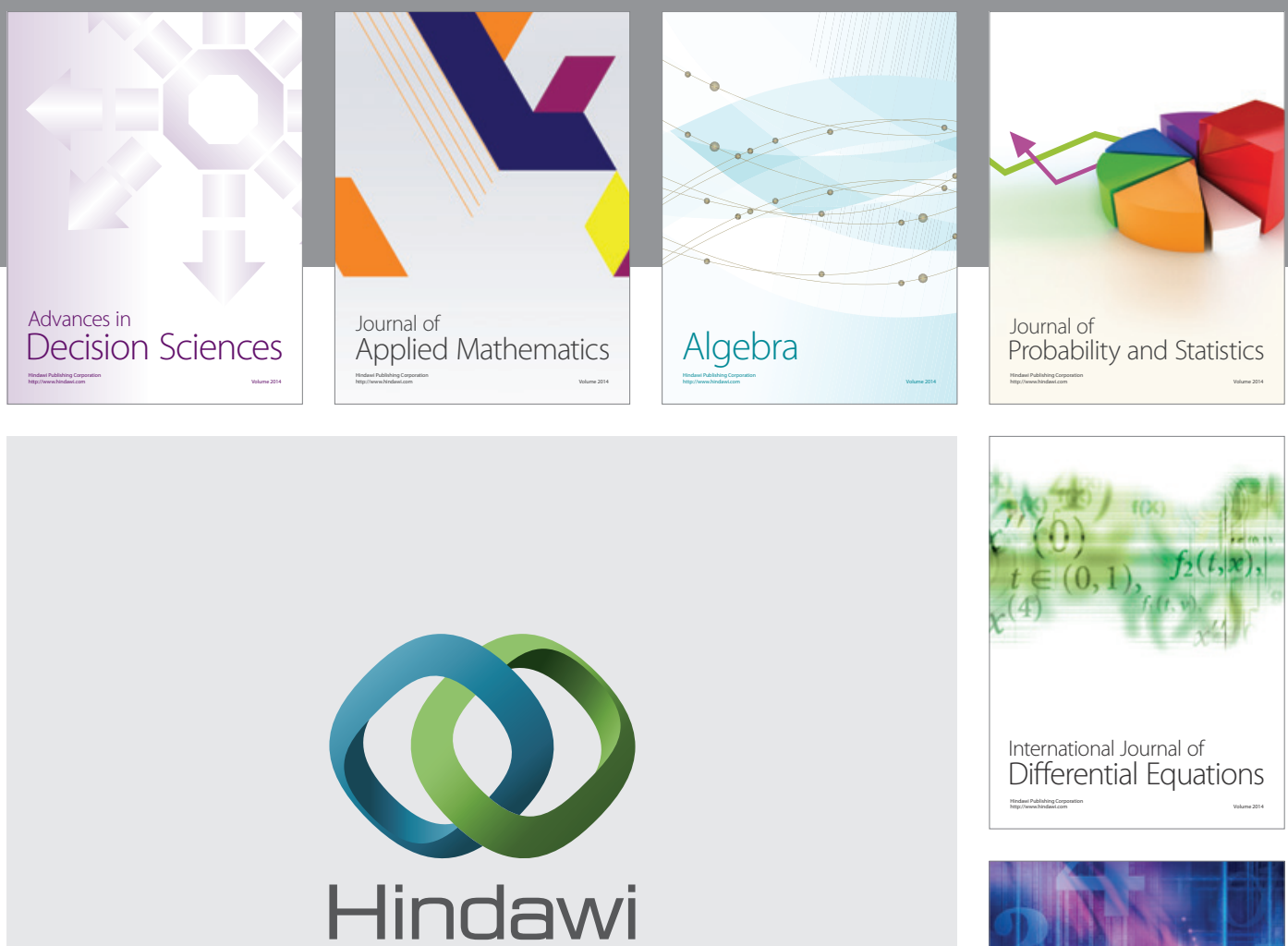

Submit your manuscripts at http://www.hindawi.com
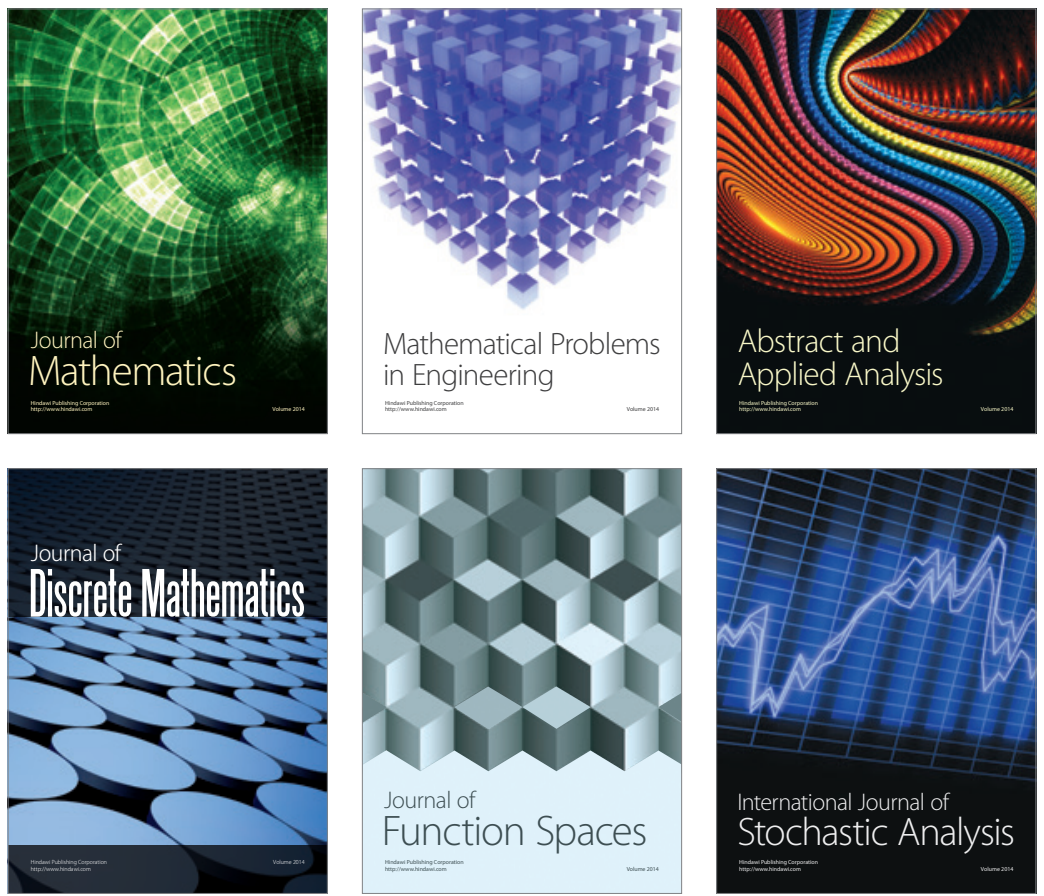

Journal of

Function Spaces

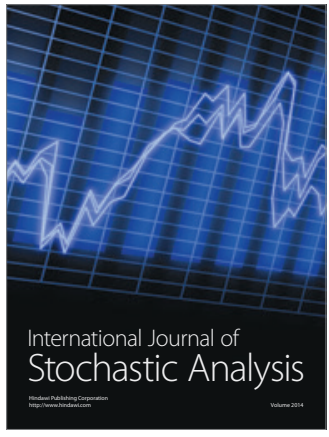

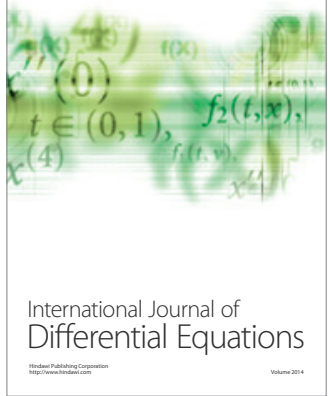
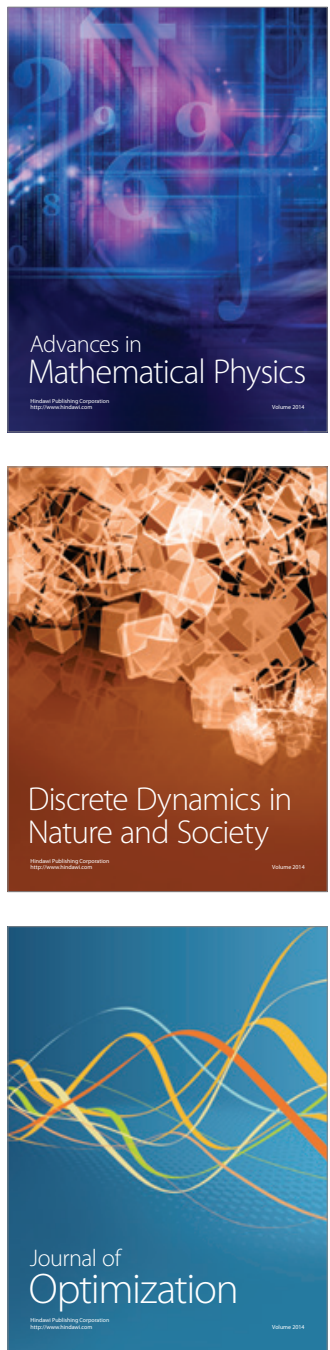